\title{
Menger curvature and Lipschitz parametrizations in metric spaces
}

\author{
by
}

\author{
Immo Hahlomaa (Jyväskylä)
}

\begin{abstract}
We show that pointwise bounds on the Menger curvature imply Lipschitz parametrization for general compact metric spaces. We also give some estimates on the optimal Lipschitz constants of the parametrizing maps for the metric spaces in $\Omega(\varepsilon)$, the class of bounded metric spaces $E$ such that the maximum angle for every triple in $E$ is at least $\pi / 2+\arcsin \varepsilon$. Finally, we extend Peter Jones's travelling salesman theorem to general metric spaces.
\end{abstract}

1. Introduction. In this paper $E$ is always a metric space and $d$ : $E \times E \rightarrow \mathbb{R}$ is a metric on $E$. We define

$$
d(E)=\sup \{d(x, y): x, y \in E\},
$$

and for $x \in E$ and $r>0$,

$$
B(x, r)=\{y \in E: d(y, x) \leq r\} .
$$

Let $\{x, y, z\}$ be three distinct points in a metric space and $i$ an isometry from $\{x, y, z\}$ to $\mathbb{R}^{2}$. For $\{x, y, z\}$ the angle at $x$, denoted by $\varangle y x z$, is the angle at vertex $i(x)$ of the planar triangle whose other vertices are $i(y)$ and $i(z)$. Using the cosine formula we can write

$$
\varangle y x z=\arccos \frac{d(x, y)^{2}+d(x, z)^{2}-d(y, z)^{2}}{2 d(x, y) d(x, z)} .
$$

We also denote the maximum angle of $\{x, y, z\}$ by $\max \varangle\{x, y, z\}$. The Menger curvature of the triple $\{x, y, z\}$, denoted by $c(x, y, z)$, is the inverse of the radius of the circle passing through $i(x), i(y)$ and $i(z)$. By elementary plane geometry

$$
c(x, y, z)=\frac{2 \sin \varangle x y z}{d(x, z)},
$$

2000 Mathematics Subject Classification: Primary 51F99; Secondary 30E20.

The author was supported by the Academy of Finland, project 23795, and by the University of Jyväskylä. 
from which we easily get

$$
c(x, y, z)=\frac{\sqrt{\left(d_{1}+d_{2}+d_{3}\right)\left(d_{1}+d_{2}-d_{3}\right)\left(d_{1}-d_{2}+d_{3}\right)\left(-d_{1}+d_{2}+d_{3}\right)}}{d_{1} d_{2} d_{3}},
$$

where $d_{1}=d(x, y), d_{2}=d(y, z)$ and $d_{3}=d(x, z)$. The condition $c(x, y, z)=0$ means that the maximum distance in $\{x, y, z\}$ is the same as the sum of the other two distances.

Karl Menger introduced this definition of curvature in [10]. In his terminology a metric space $E$ has at a point $p$ the curvature $K_{M}(p)$ if $c(x, y, z) \rightarrow$ $K_{M}(p)$ as the distinct points $x, y$ and $z$ converge independently and simultaneously to $p$. He proved that a simple metric arc $\Gamma$ such that $K_{M}(p)=0$ for all $p \in \Gamma$ and such that each subset of four points of $\Gamma$ is isometric with a subset of $\mathbb{R}^{3}$ is isometric with a segment of $\mathbb{R}$. Schoenberg showed in [12] that the latter condition in this statement can be replaced by the weaker condition that for any four points of $\Gamma$ the so-called ptolemaic inequality is satisfied.

Menger curvature has turned out to be a useful tool for studying relations between rectifiability, Cauchy integral and analytic capacity. For $z_{1}, z_{2}, z_{3}$ $\in \mathbb{C}$ we have

$$
c\left(z_{1}, z_{2}, z_{3}\right)^{2}=\sum_{\sigma} \frac{1}{\left(z_{\sigma(1)}-z_{\sigma(3)}\right) \overline{\left(z_{\sigma(2)}-z_{\sigma(3)}\right)}},
$$

where $\sigma$ runs through all six permutations of $\{1,2,3\}$. This relation between Menger curvature and the Cauchy kernel $1 / z, z \in \mathbb{C}$, was found by Melnikov in [8]. We say that $F \subset \mathbb{C}$ is 1-regular if there exists $C<\infty$ such that $C^{-1} r \leq \mathcal{H}^{1}(F \cap B(x, r)) \leq C r$ whenever $x \in F$ and $\left.r \in\right] 0, d(F)[$, where $\mathcal{H}^{1}$ is the 1-dimensional Hausdorff measure. In [7] Mattila, Melnikov and Verdera proved that for a compact 1-regular set $F \subset \mathbb{C}$ the Cauchy singular integral operator is bounded in $L^{2}(F)$ with respect to the restriction of $\mathcal{H}^{1}$ to $F$ if and only if $F$ is contained in a 1-regular curve. They first proved, by using earlier work of David and Semmes (see [4]) that the latter condition is satisfied if and only if there exists $M<\infty$ such that

$$
\iiint_{(F \cap B)^{3}} c\left(z_{1}, z_{2}, z_{3}\right)^{2} d \mathcal{H}^{1} z_{1} d \mathcal{H}^{1} z_{2} d \mathcal{H}^{1} z_{3} \leq M d(B)
$$

for every ball $B$ in $\mathbb{C}$. Using the identity (2) they obtained the final conclusion.

David and Léger have proved that if $F \subset \mathbb{C}$ with $\mathcal{H}^{1}(F)<\infty$ and

$$
\iint_{F} \int_{F} c\left(z_{1}, z_{2}, z_{3}\right)^{2} d \mathcal{H}^{1} z_{1} d \mathcal{H}^{1} z_{2} d \mathcal{H}^{1} z_{3}<\infty,
$$


then there are rectifiable curves $\Gamma_{1}, \Gamma_{2}, \ldots$ such that

$$
\mathcal{H}^{1}\left(F \backslash \bigcup_{i=1}^{\infty} \Gamma_{i}\right)=0 .
$$

We say that a set is a rectifiable curve if it is the image of a bounded interval under a Lipschitz map. Léger's proof can be found in [6]. David used this theorem when he proved in [3] that if $F \subset \mathbb{C}$ is compact with $\mathcal{H}^{1}(F)<\infty$ and $\mathcal{H}^{1}(F \cap \Gamma)=0$ for every rectifiable curve $\Gamma$, then $F$ is removable for bounded analytic functions. This last conclusion means that for every open set $U$ containing $F$ every bounded analytic function in $U \backslash F$ has an analytic extension to $U$ or, equivalently, every bounded analytic function in $\mathbb{C} \backslash F$ is constant. In [13] Tolsa proved that a compact set $F \subset \mathbb{C}$ is not removable for bounded analytic functions if and only if $F$ supports a positive Radon measure $\mu$ such that $\mu(B) \leq d(B)$ for every ball $B$ in $\mathbb{C}$ and

$$
\iiint c\left(z_{1}, z_{2}, z_{3}\right)^{2} d \mu z_{1} d \mu z_{2} d \mu z_{3}<\infty .
$$

We say that $E$ has the complete property $\Omega$ if $\max \varangle\{x, y, z\}>\pi / 2$ for every triple $\{x, y, z\} \subset E$. If there is $\alpha>0$ such that $\max \varangle\{x, y, z\} \geq$ $\pi / 2+\alpha$ for every triple $\{x, y, z\} \subset E$, we say that $E$ has the complete property $\Omega^{*}$ (with a constant $\alpha$ ). This means that

$$
d(x, z)^{2} \geq d(x, y)^{2}+d(y, z)^{2}+2 d(x, y) d(y, z) \sin \alpha
$$

for $\{x, y, z\} \subset E$ whenever $d(x, z)=d(\{x, y, z\})$. We also denote by $\Omega(\varepsilon)$, $0<\varepsilon \leq 1$, the set of bounded metric spaces which have the complete property $\Omega^{*}$ with the constant $\arcsin \varepsilon$. We say that $E$ has the property $\Omega^{*}$ at a point $x \in E$ if there exists $\delta_{x}>0$ such that $B\left(x, \delta_{x}\right)$ has the complete property $\Omega^{*}$. If $E$ has the property $\Omega^{*}$ at each of its points, we say that $E$ has the property $\Omega^{*}$.

Compact connected metric spaces with properties $\Omega$ and $\Omega^{*}$ have been studied in [2]. In this paper we prove that pointwise bounds on the Menger curvature imply Lipschitz parametrization for general compact metric spaces. We also give rather sharp estimates on the Lipschitz constants of the parametrizing maps. In Theorem 3.7 we show that for $E \in \Omega(\varepsilon)$ there exist $A \subset[0,1]$ and a surjective map $f: A \rightarrow E$ such that

for all $s, t \in A$.

$$
d(E) \frac{\varepsilon}{2}|s-t| \leq d(f(s), f(t)) \leq d(E) \frac{9}{2 \varepsilon}|s-t|
$$

For $F \subset \mathbb{R}^{n}$ and a cube $Q \subset \mathbb{R}^{n}$ set

$$
\beta_{F}(Q)=\inf _{L} d(Q)^{-1} \sup \{d(y, L): y \in F \cap 3 Q\},
$$

where the infimum is taken over all lines in $\mathbb{R}^{n}$ and $3 Q$ is the cube with the same center as $Q$ and sides parallel to the sides of $Q$, but whose diameter 
is $3 d(Q)$. A cube $Q \subset \mathbb{R}^{n}$ is dyadic if $Q=\prod_{i=1}^{n}\left[k_{i} 2^{-k},\left(k_{i}+1\right) 2^{-k}\right]$, where $k \in \mathbb{Z}$ and $k_{i} \in \mathbb{Z}$ for $i=1, \ldots n$. P. W. Jones proved in [5] that a compact $F \subset \mathbb{R}^{n}$ is contained in a rectifiable curve if

$$
\sum_{Q} \beta_{F}(Q)^{2} d(Q)<\infty
$$

where the sum is taken over all dyadic cubes in $\mathbb{R}^{n}$. F. Ferrari, B. Franchi and $\mathrm{H}$. Pajot have extended this result to geodesic metric spaces of a certain type. Theorem 5.3 is some kind of an analog in the setting of general metric spaces.

In fact, Jones proved in the case $n=2$ that for a compact $F \subset \mathbb{R}^{n}$ the condition (4) is satisfied if and only if $F$ lies in a rectifiable curve. In [11] Okikiolu extended this result to general $n \in \mathbb{N}$.

2. Order. We say that an injective map $j: E \rightarrow \mathbb{R}$ is an order on $E$ if for all $x, y, z \in E$ the condition $j(x)<j(y)<j(z)$ implies that $d(x, z)>$ $\max \{d(x, y), d(y, z)\}$.

If $j: E \rightarrow \mathbb{R}$ is an order on $E$ and $E^{\prime} \subset E$, clearly the restriction $\left.j\right|_{E^{\prime}}: E^{\prime} \rightarrow \mathbb{R}$ is an order on $E^{\prime}$. For $A \subset \mathbb{R}$ a function $j: A \rightarrow \mathbb{R}$ is an order if and only if $j$ is strictly increasing or decreasing. If $j_{1}$ and $j_{2}$ are orders on $E$ then $j_{2}=s \circ j_{1}$, where $s=j_{2} \circ j_{1}^{-1}: j_{1}(E) \rightarrow \mathbb{R}$ is an order on $j_{1}(E)$. On the other hand, if $j$ is an order on $E$ and $s: j(E) \rightarrow \mathbb{R}$ is strictly increasing or decreasing, then $s \circ j$ is also an order on $E$. If $E$ has an order, by the next proof we can construct one in the following way: Choose $a, b \in E, a \neq b$, and set, for all $x \in E$,

$$
j(x)= \begin{cases}-d(x, a) & \text { if } d(x, b)>\max \{d(x, a), d(a, b)\}, \\ d(x, a) & \text { elsewhere. }\end{cases}
$$

For $\left\{x_{1}, \ldots, x_{n}\right\} \subset E, n \in \mathbb{N}$, we will use the notation $x_{1} x_{2} \ldots x_{n}$ if there is an order $j$ on $\left\{x_{1}, \ldots, x_{n}\right\}$ such that $j\left(x_{i}\right)<j\left(x_{i+1}\right)$ for $i=1, \ldots, n-1$. In particular, $x y z$ will symbolize the relation $d(x, z)>\max \{d(x, y), d(y, z)\}$.

Proposition 2.1. Let $E$ be a metric space such that each subset of $E$ of at most four points has an order. Then the whole space $E$ has an order.

Proof. Choose $a, b \in E, a \neq b$, and define $j: E \rightarrow \mathbb{R}$ by (5). We check first that $j$ is injective. Let $x, y \in E$ with $x \neq y$. Clearly $j(x) \neq 0=j(a)$ for $x \neq a$ and $j(x) \neq j(y)$ when $d(x, a) \neq d(y, a)$. Hence we can assume that $x, y \neq a$ and $d(x, a)=d(y, a)$. Let $i$ be an order on $\{a, b, x, y\} \subset E$. Since $d(x, a)=d(y, a)$, we have either $i(x)<i(a)<i(y)$ or $i(y)<i(a)<i(x)$. We can assume that $i(x)<i(a)<i(y)$ is true. If now $i(b)<i(a)$, then $d(x, b)<$ $d(\{x, a, b\})$ and $y a b$. Thus $j(x)=d(x, a) \neq-d(x, a)=-d(y, a)=j(y)$. If $i(b)>i(a)$, we conclude similarly that $j(x)<0$ and $j(y)>0$. 
We next show that every subset of $E$ which consists of five points has an order. Let $\left\{x_{1}, x_{2}, x_{3}, x_{4}, x_{5}\right\} \subset E$ be such a set and let $i:\left\{x_{1}, x_{2}, x_{3}, x_{4}\right\} \rightarrow$ $\mathbb{R}$ be an order such that $i\left(x_{k}\right)=k$ for $k=1,2,3,4$. Choose $l, m \in\{1,2,3,4\}$, $l<m$, such that $d\left(x_{5}, x_{l}\right) \leq d\left(x_{5}, x_{n}\right)$ and $d\left(x_{5}, x_{m}\right) \leq d\left(x_{5}, x_{n}\right)$ for $n \in$ $\{1,2,3,4\} \backslash\{l, m\}$. Then $m=l+1$. Indeed, otherwise $l<n<m$ with some integer $n$ and $x_{l} x_{n} x_{m}$. Therefore for any order $i^{\prime}$ on $\left\{x_{l}, x_{n}, x_{m}, x_{5}\right\}$ either $i^{\prime}\left(x_{l}\right)<i^{\prime}\left(x_{n}\right)<i^{\prime}\left(x_{m}\right)$ or $i^{\prime}\left(x_{m}\right)<i^{\prime}\left(x_{n}\right)<i^{\prime}\left(x_{l}\right)$. Thus $d\left(x_{5}, x_{n}\right)<$ $\max \left\{d\left(x_{5}, x_{l}\right), d\left(x_{5}, x_{m}\right)\right\}$, which contradicts the choice of $l$ and $m$.

If $x_{5} x_{l} x_{l+1}$, set $p=l-1 / 2$. If $x_{l} x_{5} x_{l+1}$, set $p=l+1 / 2$. Finally, if $x_{5} x_{l+1} x_{l}$, set $p=l+3 / 2$. Define $h:\left\{x_{1}, x_{2}, x_{3}, x_{4}, x_{5}\right\} \rightarrow \mathbb{R}$ by setting $h\left(x_{k}\right)=i\left(x_{k}\right)$ $=k$ for $k=1,2,3,4$ and $h\left(x_{5}\right)=p$. We claim that $h$ is an order. Clearly $h$ is injective. We have to show that for every triple $\{k, m, n\} \subset\{1,2,3,4,5\}$ the condition $h\left(x_{k}\right)<h\left(x_{m}\right)<h\left(x_{n}\right)$ implies that $x_{k} x_{m} x_{n}$. For $\{l, l+1,5\}$ this is true by the definition of $h$. Obviously, it suffices to check the triples of indices which contain 5 .

If $l=1$, then $h\left(x_{5}\right) \leq l+3 / 2<h\left(x_{3}\right)<h\left(x_{4}\right)$. Since $i$ is an order on $\left\{x_{1}, x_{2}, x_{3}, x_{4}\right\}$, we have $x_{1} x_{3} x_{4}$. Thus for any order $i^{\prime}$ on $\left\{x_{1}, x_{3}, x_{4}, x_{5}\right\}$ either $i^{\prime}\left(x_{1}\right)<i^{\prime}\left(x_{3}\right)<i^{\prime}\left(x_{4}\right)$ or $i^{\prime}\left(x_{4}\right)<i^{\prime}\left(x_{3}\right)<i^{\prime}\left(x_{1}\right)$. Since $d\left(x_{5}, x_{1}\right) \leq$ $d\left(x_{5}, x_{3}\right)$ and $i^{\prime}$ is an order, necessarily $x_{5} x_{3} x_{4}$.

If $l=2$, then $h\left(x_{1}\right)<l-1 / 2 \leq h\left(x_{5}\right) \leq l+3 / 2<h\left(x_{4}\right)$. Since $i$ is an order on $\left\{x_{1}, x_{2}, x_{3}, x_{4}\right\}$, we have $x_{1} x_{2} x_{4}$. Thus for any order $i^{\prime}$ on $\left\{x_{1}, x_{2}, x_{4}, x_{5}\right\}$ either $i^{\prime}\left(x_{1}\right)<i^{\prime}\left(x_{2}\right)<i^{\prime}\left(x_{4}\right)$ or $i^{\prime}\left(x_{4}\right)<i^{\prime}\left(x_{2}\right)<i^{\prime}\left(x_{1}\right)$. Since $d\left(x_{5}, x_{2}\right) \leq d\left(x_{5}, x_{1}\right)$ and $d\left(x_{5}, x_{2}\right) \leq d\left(x_{5}, x_{4}\right)$ and $i^{\prime}$ is an order, necessarily $x_{1} x_{5} x_{4}$.

If $l=3$, then $h\left(x_{5}\right) \geq l-1 / 2>h\left(x_{2}\right)>h\left(x_{1}\right)$. Since $i$ is an order on $\left\{x_{1}, x_{2}, x_{3}, x_{4}\right\}$, we have $x_{1} x_{2} x_{3}$. Thus for any order $i^{\prime}$ on $\left\{x_{1}, x_{2}, x_{3}, x_{5}\right\}$ either $i^{\prime}\left(x_{1}\right)<i^{\prime}\left(x_{2}\right)<i^{\prime}\left(x_{3}\right)$ or $i^{\prime}\left(x_{3}\right)<i^{\prime}\left(x_{2}\right)<i^{\prime}\left(x_{1}\right)$. Since $d\left(x_{5}, x_{3}\right) \leq$ $d\left(x_{5}, x_{2}\right)$ and $i^{\prime}$ is an order, necessarily $x_{5} x_{2} x_{1}$.

Suppose that $l \leq 2, k \in\{l+2,4\}$ and we have $x_{5} x_{l} x_{l+1}$ or $x_{l} x_{5} x_{l+1}$. Then $h\left(x_{5}\right) \leq l+1 / 2<h\left(x_{l+1}\right)<h\left(x_{k}\right)$. Since $i$ is an order on $\left\{x_{1}, x_{2}\right.$, $\left.x_{3}, x_{4}\right\}$, we have $x_{l} x_{l+1} x_{k}$. Thus for any order $i^{\prime}$ on $\left\{x_{l}, x_{l+1}, x_{k}, x_{5}\right\}$ either $i^{\prime}\left(x_{l}\right)<i^{\prime}\left(x_{l+1}\right)<i^{\prime}\left(x_{k}\right)$ or $i^{\prime}\left(x_{k}\right)<i^{\prime}\left(x_{l+1}\right)<i^{\prime}\left(x_{l}\right)$. Since $d\left(x_{5}, x_{l}\right)<$ $d\left(\left\{x_{5}, x_{l+1}, x_{l}\right\}\right)$ and $i^{\prime}$ is an order, necessarily $x_{5} x_{l+1} x_{k}$.

Suppose that $l \leq 2, k \in\{l+2,4\}$ and we have $x_{l} x_{5} x_{l+1}$ or $x_{5} x_{l+1} x_{l}$. Then $h\left(x_{l}\right)<l+1 / 2 \leq h\left(x_{5}\right) \leq l+3 / 2<h\left(x_{k}\right)$. Since $i$ is an order on $\left\{x_{1}, x_{2}, x_{3}, x_{4}\right\}$, we have $x_{l} x_{l+1} x_{k}$. Thus for any order $i^{\prime}$ on $\left\{x_{l}, x_{l+1}, x_{k}, x_{5}\right\}$ either $i^{\prime}\left(x_{l}\right)<i^{\prime}\left(x_{l+1}\right)<i^{\prime}\left(x_{k}\right)$ or $i^{\prime}\left(x_{k}\right)<i^{\prime}\left(x_{l+1}\right)<i^{\prime}\left(x_{l}\right)$. Since $d\left(x_{5}, x_{l+1}\right)$ $<d\left(\left\{x_{5}, x_{l}, x_{l+1}\right\}\right)$ and $d\left(x_{5}, x_{l+1}\right) \leq d\left(x_{5}, x_{k}\right)$ and $i^{\prime}$ is an order, necessarily $x_{l} x_{5} x_{k}$.

Suppose that $l \leq 2, k \in\{l+2,4\}$ and $x_{5} x_{l} x_{l+1}$. Then $h\left(x_{5}\right)<h\left(x_{l}\right)<$ $h\left(x_{k}\right)$. Since $i$ is an order on $\left\{x_{1}, x_{2}, x_{3}, x_{4}\right\}$, we have $x_{l} x_{l+1} x_{k}$. Thus for 
any order $i^{\prime}$ on $\left\{x_{l}, x_{l+1}, x_{k}, x_{5}\right\}$ either $i^{\prime}\left(x_{l}\right)<i^{\prime}\left(x_{l+1}\right)<i^{\prime}\left(x_{k}\right)$ or $i^{\prime}\left(x_{k}\right)<$ $i^{\prime}\left(x_{l+1}\right)<i^{\prime}\left(x_{l}\right)$. Since $x_{5} x_{l} x_{l+1}$ and $i^{\prime}$ is an order, necessarily $x_{5} x_{l} x_{k}$.

Suppose that $l \leq 2, k \in\{l+2,4\}$ and $x_{5} x_{l+1} x_{l}$. Then $h\left(x_{l+1}\right)<h\left(x_{5}\right)=$ $l+3 / 2<h\left(x_{k}\right)$. Since $i$ is an order on $\left\{x_{1}, x_{2}, x_{3}, x_{4}\right\}$, we have $x_{l} x_{l+1} x_{k}$. Thus for any order $i^{\prime}$ on $\left\{x_{l}, x_{l+1}, x_{k}, x_{5}\right\}$ either $i^{\prime}\left(x_{l}\right)<i^{\prime}\left(x_{l+1}\right)<i^{\prime}\left(x_{k}\right)$ or $i^{\prime}\left(x_{k}\right)<i^{\prime}\left(x_{l+1}\right)<i^{\prime}\left(x_{l}\right)$. Since $d\left(x_{5}, x_{l+1}\right) \leq d\left(x_{5}, x_{k}\right), x_{5} x_{l+1} x_{l}$ and $i^{\prime}$ is an order, necessarily $x_{l+1} x_{5} x_{k}$.

Suppose that $l \geq 2, k \in\{1, l-1\}$ and we have $x_{l} x_{5} x_{l+1}$ or $x_{5} x_{l+1} x_{l}$. Then $h\left(x_{5}\right) \geq l+1 / 2>h\left(x_{l}\right)>h\left(x_{k}\right)$. Since $i$ is an order on $\left\{x_{1}, x_{2}, x_{3}, x_{4}\right\}$, we have $x_{k} x_{l} x_{l+1}$. Thus for any order $i^{\prime}$ on $\left\{x_{k}, x_{l}, x_{l+1}, x_{5}\right\}$ either $i^{\prime}\left(x_{k}\right)<i^{\prime}\left(x_{l}\right)<i^{\prime}\left(x_{l+1}\right)$ or $i^{\prime}\left(x_{l+1}\right)<i^{\prime}\left(x_{l}\right)<i^{\prime}\left(x_{k}\right)$. Since $d\left(x_{5}, x_{l+1}\right)$ $<d\left(\left\{x_{5}, x_{l}, x_{l+1}\right\}\right)$ and $i^{\prime}$ is an order, necessarily $x_{5} x_{l} x_{k}$.

Suppose that $l \geq 2, k \in\{1, l-1\}$ and we have $x_{5} x_{l} x_{l+1}$ or $x_{l} x_{5} x_{l+1}$. Then $h\left(x_{l+1}\right)>l+1 / 2 \geq h\left(x_{5}\right) \geq l-1 / 2>h\left(x_{k}\right)$. Since $i$ is an order on $\left\{x_{1}, x_{2}, x_{3}, x_{4}\right\}$, we have $x_{k} x_{l} x_{l+1}$. Thus for any order $i^{\prime}$ on $\left\{x_{k}, x_{l}, x_{l+1}, x_{5}\right\}$ either $i^{\prime}\left(x_{k}\right)<i^{\prime}\left(x_{l}\right)<i^{\prime}\left(x_{l+1}\right)$ or $i^{\prime}\left(x_{l+1}\right)<i^{\prime}\left(x_{l}\right)<i^{\prime}\left(x_{k}\right)$. Since $d\left(x_{5}, x_{l}\right)$ $<d\left(x_{5}, x_{l+1}, x_{l}\right)$ and $d\left(x_{5}, x_{l}\right) \leq d\left(x_{5}, x_{k}\right)$ and $i^{\prime}$ is an order, necessarily $x_{k} x_{5} x_{l+1}$.

Suppose that $l \geq 2, k \in\{1, l-1\}$ and $x_{5} x_{l+1} x_{l}$. Then $h\left(x_{k}\right)<h\left(x_{l+1}\right)<$ $h\left(x_{5}\right)$. Since $i$ is an order on $\left\{x_{1}, x_{2}, x_{3}, x_{4}\right\}$, we have $x_{k} x_{l} x_{l+1}$. Thus for any order $i^{\prime}$ on $\left\{x_{k}, x_{l}, x_{l+1}, x_{5}\right\}$ either $i^{\prime}\left(x_{k}\right)<i^{\prime}\left(x_{l}\right)<i^{\prime}\left(x_{l+1}\right)$ or $i^{\prime}\left(x_{l+1}\right)<$ $i^{\prime}\left(x_{l}\right)<i^{\prime}\left(x_{k}\right)$. Since $x_{5} x_{l+1} x_{l}$ and $i^{\prime}$ is an order, necessarily $x_{5} x_{l+1} x_{k}$.

Suppose that $l \geq 2, k \in\{1, l-1\}$ and $x_{5} x_{l} x_{l+1}$. Then $h\left(x_{k}\right)<h\left(x_{5}\right)<$ $h\left(x_{l}\right)$. Since $i$ is an order on $\left\{x_{1}, x_{2}, x_{3}, x_{4}\right\}$, we have $x_{k} x_{l} x_{l+1}$. Thus for any order $i^{\prime}$ on $\left\{x_{k}, x_{l}, x_{l+1}, x_{5}\right\}$ either $i^{\prime}\left(x_{k}\right)<i^{\prime}\left(x_{l}\right)<i^{\prime}\left(x_{l+1}\right)$ or $i^{\prime}\left(x_{l+1}\right)<$ $i^{\prime}\left(x_{l}\right)<i^{\prime}\left(x_{k}\right)$. Since $x_{5} x_{l} x_{l+1}$ and $d\left(x_{5}, x_{l}\right) \leq d\left(x_{5}, x_{k}\right)$ and $i^{\prime}$ is an order, necessarily $x_{k} x_{5} x_{l}$.

So we have shown that every subset of $E$ which consists of five points has an order. Let now $x_{1}, x_{2}, x_{3} \in E$ and $0 \leq j\left(x_{1}\right)<j\left(x_{2}\right)<j\left(x_{3}\right)$. Let $i$ be an order on $\left\{a, b, x_{1}, x_{2}, x_{3}\right\}$ such that $i(a)<i(b)$. Since $d\left(x_{k}, b\right) \leq$ $\max \left\{d\left(x_{k}, a\right), d(a, b)\right\}$ for $k=1,2,3$, necessarily $i\left(x_{k}\right) \geq i(a)$ for $k=1,2,3$. Since $d\left(x_{1}, a\right)<d\left(x_{2}, a\right)<d\left(x_{3}, a\right)$, we further have $i\left(x_{1}\right)<i\left(x_{2}\right)<i\left(x_{3}\right)$. This implies that $x_{1} x_{2} x_{3}$.

Let next $x_{1}, x_{2}, x_{3} \in E$ and $j\left(x_{1}\right)<0 \leq j\left(x_{2}\right)<j\left(x_{3}\right)$ and let $i$ be an order on $\left\{a, b, x_{1}, x_{2}, x_{3}\right\}$ such that $i(a)<i(b)$. Since $d\left(x_{k}, b\right) \leq$ $\max \left\{d\left(x_{k}, a\right), d(a, b)\right\}$ for $k=2,3$, necessarily $i\left(x_{k}\right) \geq i(a)$ for $k=2,3$. Since $d\left(x_{2}, a\right)<d\left(x_{3}, a\right)$, we have $i\left(x_{2}\right)<i\left(x_{3}\right)$. Moreover, $i\left(x_{1}\right)<i(a)$, because $x_{1} a b$. So $i\left(x_{1}\right)<i(a) \leq i\left(x_{2}\right)<i\left(x_{3}\right)$, which implies $x_{1} x_{2} x_{3}$.

Let next $x_{1}, x_{2}, x_{3} \in E$ and $j\left(x_{1}\right)<j\left(x_{2}\right)<0 \leq j\left(x_{3}\right)$ and let $i$ be an order on $\left\{a, b, x_{1}, x_{2}, x_{3}\right\}$ such that $i(a)<i(b)$. Since $x_{k} a b$ for $k=1,2$, necessarily $i\left(x_{k}\right)<i(a)$ for $k=1,2$. Since $d\left(x_{2}, a\right)<d\left(x_{1}, a\right)$, we have $i\left(x_{1}\right)<$ 
$i\left(x_{2}\right)$. Moreover $i\left(x_{3}\right) \geq i(a)$, because $d\left(x_{3}, b\right) \leq \max \left\{d\left(x_{3}, a\right), d(a, b)\right\}$. So $i\left(x_{1}\right)<i\left(x_{2}\right)<i(a) \leq i\left(x_{3}\right)$, which implies $x_{1} x_{2} x_{3}$.

Finally, let $x_{1}, x_{2}, x_{3} \in E$ and $j\left(x_{1}\right)<j\left(x_{2}\right)<j\left(x_{3}\right)<0$ and let $i$ be an order on $\left\{a, b, x_{1}, x_{2}, x_{3}\right\}$ such that $i(a)<i(b)$. Since $x_{k} a b$ for $k=1,2,3$, necessarily $i\left(x_{k}\right)<i(a)$ for $k=1,2,3$. Since $d\left(x_{3}, a\right)<d\left(x_{2}, a\right)<d\left(x_{1}, a\right)$, we have $i\left(x_{1}\right)<i\left(x_{2}\right)<i\left(x_{3}\right)$. This implies $x_{1} x_{2} x_{3}$.

The following two lemmas will be used in Section 5 .

Lemma 2.2. Let $K \geq 1$ and $\varepsilon \geq K /(K+1)$. Suppose that $E$ is a metric space of four points such that $d(x, y)<K d(z, w)$ for all $x, y, z, w \in E$, $z \neq w$, and $d(x, z) \geq d(x, y)+\varepsilon d(y, z)$ whenever $x, y, z \in E$ are such that $d(x, z)=d(\{x, y, z\})$. Then either $E$ has an order or $E=\left\{x_{1}, x_{2}, x_{3}, x_{4}\right\}$, where $x_{1} x_{2} x_{3}, x_{2} x_{1} x_{4}, x_{2} x_{3} x_{4}, x_{1} x_{4} x_{3}, \varepsilon d\left(x_{1}, x_{2}\right) \leq d\left(x_{3}, x_{4}\right) \leq \varepsilon^{-1} d\left(x_{1}, x_{2}\right)$ and $\varepsilon d\left(x_{1}, x_{4}\right) \leq d\left(x_{2}, x_{3}\right) \leq \varepsilon^{-1} d\left(x_{1}, x_{4}\right)$.

Proof. Let $E=\left\{x_{1}, x_{2}, x_{3}, x_{4}\right\}$ be such that $x_{1} x_{2} x_{3}$. We define $\delta=$ $d(E) / K$ and $d_{i j}=d\left(x_{i}, x_{j}\right)$ for $i, j=1, \ldots, 4$. If $x_{i} x_{j} x_{k}$, we have $d_{i j} \leq$ $d_{i k}-\varepsilon d_{j k}<\delta(K-\varepsilon)$.

Suppose first that $x_{1} x_{2} x_{4}$ and $x_{1} x_{3} x_{4}$. Then $d_{24}-d_{23} \geq d_{14}-d_{12}-d_{23} \geq$ $d_{13}+\varepsilon d_{34}-d_{12}-d_{23} \geq d_{12}+\varepsilon d_{23}+\varepsilon d_{34}-d_{12}-d_{23}>\delta((\varepsilon-1) K+\varepsilon)$ $\geq 0$ and $d_{24}-d_{34} \geq d_{14}-d_{12}-\left(d_{14}-\varepsilon d_{13}\right) \geq \varepsilon\left(d_{12}+\varepsilon d_{23}\right)-d_{12}>$ $\delta\left((\varepsilon-1)(K-\varepsilon)+\varepsilon^{2}\right)=\delta((\varepsilon-1) K+\varepsilon) \geq 0$. Thus we have $x_{1} x_{2} x_{3} x_{4}$.

If $x_{1} x_{2} x_{4}$ and $x_{1} x_{4} x_{3}$, then $d_{23}-d_{24} \geq d_{23}-\left(d_{14}-\varepsilon d_{12}\right) \geq d_{23}-\left(d_{12}+\right.$ $\left.d_{23}-\varepsilon d_{34}-\varepsilon d_{12}\right)>\delta((\varepsilon-1) K+\varepsilon) \geq 0$ and $d_{23}-d_{34} \geq d_{23}-\left(d_{12}+d_{23}-\right.$ $\left.\varepsilon d_{14}\right) \geq \varepsilon\left(d_{12}+\varepsilon d_{24}\right)-d_{12}>\delta\left((\varepsilon-1)(K-\varepsilon)+\varepsilon^{2}\right) \geq 0$, which implies $x_{1} x_{2} x_{4} x_{3}$.

If $x_{1} x_{4} x_{2}$ and $x_{1} x_{4} x_{3}$, then $d_{34}-d_{24} \geq d_{12}+\varepsilon d_{23}-d_{14}-\left(d_{12}-\varepsilon d_{14}\right)>$ $\delta((\varepsilon-1) K+\varepsilon) \geq 0$ and $d_{34}-d_{23} \geq d_{12}+\varepsilon d_{23}-d_{14}-d_{23} \geq d_{14}+\varepsilon d_{24}+$ $\varepsilon d_{23}-d_{14}-d_{23}>\delta((\varepsilon-1) K+\varepsilon) \geq 0$, which implies $x_{1} x_{4} x_{2} x_{3}$.

If $x_{2} x_{1} x_{4}$ and $x_{3} x_{1} x_{4}$, then $d_{34}-d_{24} \geq d_{13}+\varepsilon d_{14}-\left(d_{12}+d_{14}\right) \geq$ $d_{12}+\varepsilon d_{23}+\varepsilon d_{14}-\left(d_{12}+d_{14}\right)>\delta((\varepsilon-1) K+\varepsilon) \geq 0$ and $d_{34}-d_{23} \geq$ $d_{12}+\varepsilon d_{23}+\varepsilon d_{14}-d_{23}>\delta((\varepsilon-1) K+1+\varepsilon)>0$, which implies $x_{4} x_{1} x_{2} x_{3}$.

Assume now that $x_{2} x_{1} x_{4}$ and $x_{1} x_{4} x_{3}$. Since $d_{24}+\varepsilon d_{34}-d_{23} \geq d_{12}+\varepsilon d_{14}+$ $\varepsilon\left(d_{12}+\varepsilon d_{23}-d_{14}\right)-d_{23}>\delta\left(\left(\varepsilon^{2}-1\right) K+1+\varepsilon\right)=\delta((\varepsilon-1) K+1)(1+\varepsilon)>0$ and $d_{24}+\varepsilon d_{23}-d_{34} \geq d_{12}+\varepsilon d_{14}+\varepsilon d_{23}-\left(d_{12}+d_{23}-\varepsilon d_{14}\right)>\delta((\varepsilon-1) K+2 \varepsilon)>0$, we must have $x_{2} x_{3} x_{4}$. Now $d_{24}=d_{34}+\varepsilon_{1} d_{23}=d_{12}+\varepsilon_{2} d_{14}$ and $d_{34}=$ $d_{12}+\varepsilon_{4} d_{23}-\varepsilon_{3} d_{14}$ for some $\varepsilon \leq \varepsilon_{1}, \varepsilon_{2}, \varepsilon_{3}, \varepsilon_{4} \leq 1$. This gives $\left(\varepsilon_{2}+\varepsilon_{3}\right) d_{14}=$ $\left(\varepsilon_{1}+\varepsilon_{4}\right) d_{23}$, from which we get $\varepsilon d_{14} \leq d_{23} \leq \varepsilon^{-1} d_{14}$.

No other alternatives are possible because of the triangle inequality. Namely, $x_{1} x_{2} x_{4}$ and $x_{3} x_{1} x_{4}$ would imply $d_{34}-d_{24}-d_{23} \geq d_{12}+\varepsilon d_{23}+$ $\varepsilon d_{14}-\left(d_{14}-\varepsilon d_{12}\right)-d_{23}>\delta(2(\varepsilon-1) K+1+\varepsilon) \geq 0$. If $x_{1} x_{4} x_{2}$ and $x_{1} x_{3} x_{4}$, then $d_{23}-d_{24}-d_{34} \geq d_{23}-\left(d_{12}-\varepsilon d_{14}\right)-\left(d_{14}-\varepsilon\left(d_{12}+\varepsilon d_{23}\right)\right)>$ $\delta\left(2(\varepsilon-1) K+1+\varepsilon^{2}\right) \geq 0$. If $x_{1} x_{4} x_{2}$ and $x_{3} x_{1} x_{4}$, then $d_{34}-d_{24}-d_{23} \geq$ 
$d_{12}+\varepsilon d_{23}+\varepsilon d_{14}-\left(d_{12}-\varepsilon d_{14}\right)-d_{23}>\delta((\varepsilon-1) K+2 \varepsilon)>0$. If $x_{2} x_{1} x_{4}$ and $x_{1} x_{3} x_{4}$, then $d_{24}-d_{34}-d_{23} \geq d_{14}+\varepsilon d_{12}-\left(d_{14}-\varepsilon\left(d_{12}+\varepsilon d_{23}\right)\right)-d_{23}>$ $\delta\left(\left(\varepsilon^{2}-1\right) K+2 \varepsilon\right) \geq 0$.

Lemma 2.3. Let $K \geq 1$ and $\varepsilon^{3} \geq(4 K-1) /(4 K+1)$. Suppose that $E$ is a metric space such that $\# E \neq 4, d(x, y)<K d(z, w)$ for all $x, y, z, w \in E$, $z \neq w$, and $d(x, z) \geq d(x, y)+\varepsilon d(y, z)$ whenever $x, y, z \in E$ are such that $d(x, z)=d(\{x, y, z\})$. Then $E$ has an order.

Proof. We assume that there are at least five points in $E$. We need to show that every quadruple of $E$ has an order. Suppose that this is not true and let $\left\{x_{1}, x_{2}, x_{3}, x_{4}, x_{5}\right\} \subset E$ be a subset of five points such that $\left\{x_{1}, x_{2}, x_{3}, x_{4}\right\}$ has no order. By the previous lemma we can assume that $x_{1} x_{2} x_{3}, x_{2} x_{1} x_{4}, x_{2} x_{3} x_{4}$ and $x_{1} x_{4} x_{3}$. We set $\delta=d(E) / K$ and $d_{i j}=d\left(x_{i}, x_{j}\right)$ for $i, j=1, \ldots, 4$. If $x_{i} x_{j} x_{k}$, we have $d_{i j} \leq d_{i k}-\varepsilon d_{j k}<\delta(K-\varepsilon)$.

Applying the proof of Lemma 2.2 to the quadruples $\left\{x_{1}, x_{2}, x_{3}, x_{5}\right\}$ and $\left\{x_{1}, x_{4}, x_{3}, x_{5}\right\}$, we see that the following eight cases are not possible:

$\begin{array}{lll}x_{1} x_{2} x_{5} & \text { and } & x_{3} x_{1} x_{5}, \\ x_{1} x_{5} x_{2} & \text { and } & x_{1} x_{3} x_{5}, \\ x_{1} x_{5} x_{2} & \text { and } & x_{3} x_{1} x_{5}, \\ x_{2} x_{1} x_{5} & \text { and } & x_{1} x_{3} x_{5}, \\ x_{1} x_{4} x_{5} & \text { and } & x_{3} x_{1} x_{5}, \\ x_{1} x_{5} x_{4} & \text { and } & x_{1} x_{3} x_{5}, \\ x_{1} x_{5} x_{4} & \text { and } & x_{3} x_{1} x_{5}, \\ x_{4} x_{1} x_{5} & \text { and } & x_{1} x_{3} x_{5} .\end{array}$

Furthermore, $x_{2} x_{1} x_{5}$ and $x_{1} x_{5} x_{3}$ implies $\varepsilon d_{15} \leq d_{23} \leq \varepsilon^{-1} d_{15}$. Similarly, if $x_{4} x_{1} x_{5}$ and $x_{1} x_{5} x_{3}$, we have $\varepsilon d_{15} \leq d_{34} \leq \varepsilon^{-1} d_{15}$.

The next three alternatives are not possible by the triangle inequality: If $x_{1} x_{5} x_{2}$ and $x_{1} x_{5} x_{4}$, then $d_{24}-d_{25}-d_{45} \geq d_{12}+\varepsilon d_{14}-\left(d_{12}-\varepsilon d_{15}\right)-$ $\left(d_{14}-\varepsilon d_{15}\right)>\delta((\varepsilon-1) K+2 \varepsilon)>0$. Similarly, if $x_{1} x_{5} x_{2}$ and $x_{1} x_{4} x_{5}$, then $d_{24}-d_{25}-d_{45} \geq d_{12}+\varepsilon d_{14}-\left(d_{12}-\varepsilon d_{15}\right)-\left(d_{15}-\varepsilon d_{14}\right)>\delta((\varepsilon-1) K+2 \varepsilon)>0$ and if $x_{1} x_{2} x_{5}$ and $x_{1} x_{5} x_{4}$, we have $d_{24}-d_{25}-d_{45} \geq d_{14}+\varepsilon d_{12}-\left(d_{15}-\right.$ $\left.\varepsilon d_{12}\right)-\left(d_{14}-\varepsilon d_{15}\right)>\delta((\varepsilon-1) K+2 \varepsilon)>0$. The alternative $x_{2} x_{1} x_{5}$ and $x_{4} x_{1} x_{5}$ is impossible, because in that case $d_{24}+\varepsilon d_{25}-d_{45} \geq d_{14}+\varepsilon d_{12}+$ $\varepsilon\left(d_{15}+\varepsilon d_{12}\right)-\left(d_{14}+d_{15}\right)>\delta\left((\varepsilon-1) K+\varepsilon+\varepsilon^{2}\right)>0, d_{24}+\varepsilon d_{45}-d_{25} \geq$ $d_{12}+\varepsilon d_{14}+\varepsilon\left(d_{15}+\varepsilon d_{14}\right)-\left(d_{12}+d_{15}\right)>\delta\left((\varepsilon-1) K+\varepsilon+\varepsilon^{2}\right)>0$ and $d_{25}+\varepsilon d_{45}-d_{24} \geq d_{12}+\varepsilon d_{15}+\varepsilon\left(d_{14}+\varepsilon d_{15}\right)-\left(d_{12}+d_{14}\right)>\delta\left((\varepsilon-1) K+\varepsilon+\varepsilon^{2}\right)$ $>0$.

By the above examination not more than the following six cases are possible: 


$$
\begin{array}{lll}
x_{2} x_{1} x_{5}, & x_{1} x_{5} x_{3}, & x_{1} x_{4} x_{5}, \\
x_{2} x_{1} x_{5}, & x_{1} x_{5} x_{3}, & x_{1} x_{5} x_{4}, \\
x_{1} x_{2} x_{5}, & x_{1} x_{5} x_{3}, & x_{4} x_{1} x_{5}, \\
x_{1} x_{5} x_{2}, & x_{1} x_{5} x_{3}, & x_{4} x_{1} x_{5}, \\
x_{1} x_{2} x_{5}, & x_{1} x_{3} x_{5}, & x_{1} x_{4} x_{5}, \\
x_{1} x_{2} x_{5}, & x_{1} x_{5} x_{3}, & x_{1} x_{4} x_{5} .
\end{array}
$$

From (6) it follows that $d_{45} \leq d_{15}-\varepsilon d_{14} \leq d_{15}-\varepsilon^{2} d_{23} \leq d_{15}-\varepsilon^{3} d_{15}<$ $\left(1-\varepsilon^{3}\right)(K-\varepsilon) \delta \leq \delta$, which is a contradiction. In the case $(7)$ we would have $d_{45} \leq d_{14}-\varepsilon d_{15} \leq d_{14}-\varepsilon^{2} d_{23} \leq d_{14}-\varepsilon^{3} d_{14}<\delta$. Similarly, in the case (8), $d_{25} \leq d_{15}-\varepsilon d_{12} \leq d_{15}-\varepsilon^{2} d_{34} \leq d_{15}-\varepsilon^{3} d_{15}<\delta$, and (9) would imply $d_{25} \leq d_{12}-\varepsilon d_{15} \leq d_{12}-\varepsilon^{2} d_{34} \leq d_{12}-\varepsilon^{3} d_{12}<\delta$. Thus we must have (10) or (11). Since $d_{24}+\varepsilon d_{25}-d_{45} \geq d_{12}+\varepsilon d_{14}+\varepsilon\left(d_{15}-d_{12}\right)-\left(d_{15}-\varepsilon d_{14}\right)>$ $\delta((\varepsilon-1) K+1+\varepsilon)>0$ and $d_{24}+\varepsilon d_{45}-d_{25} \geq d_{12}+\varepsilon d_{14}+\varepsilon\left(d_{15}-d_{14}\right)-$ $\left(d_{15}-\varepsilon d_{12}\right)>\delta((\varepsilon-1) K+1+\varepsilon)>0$, we further have $x_{2} x_{5} x_{4}$. Thus $d_{12}+\varepsilon_{1} d_{14}=d_{24}=d_{45}+\varepsilon_{2} d_{25}=d_{15}-\varepsilon_{3} d_{14}+\varepsilon_{2}\left(d_{15}-\varepsilon_{4} d_{12}\right)$ for some $\varepsilon \leq \varepsilon_{1}, \varepsilon_{2}, \varepsilon_{3}, \varepsilon_{4} \leq 1$, from which we get

$$
\varepsilon\left(d_{12}+d_{14}\right) \leq d_{15} \leq \frac{2\left(d_{12}+d_{14}\right)}{1+\varepsilon} .
$$

Now it follows from (10) that $d_{35} \leq d_{15}-\varepsilon d_{13} \leq 2(1+\varepsilon)^{-1}\left(d_{12}+d_{14}\right)-$ $\varepsilon\left(d_{12}+\varepsilon d_{23}\right) \leq 2(1+\varepsilon)^{-1}\left(d_{12}+d_{14}\right)-\varepsilon\left(d_{12}+\varepsilon^{2} d_{14}\right)<\left(4(1+\varepsilon)^{-1}-\varepsilon-\varepsilon^{3}\right)$ $(K-\varepsilon) \delta \leq\left(4(1+\varepsilon)^{-1}-2 \varepsilon^{2}\right)(K-\varepsilon) \delta \leq \delta$, and (11) yields $d_{35} \leq d_{13}-\varepsilon d_{15} \leq$ $d_{12}+d_{23}-\varepsilon^{2}\left(d_{12}+d_{14}\right) \leq d_{12}+d_{23}-\varepsilon^{2}\left(d_{12}+\varepsilon d_{23}\right)<\left(2-\varepsilon^{2}-\varepsilon^{3}\right)(K-\varepsilon) \delta$ $\leq \delta$.

If $c(x, y, z)=0$ for every triple $\{x, y, z\} \subset E$, we can apply the previous lemmas to finite subsets of $E$. Further by using Proposition 2.1 we easily get the following result.

Proposition 2.4. Let $E$ be a metric space such that $c(x, y, z)=0$ for any pairwise distinct points $x, y, z \in E$. Then $E$ is isometric with a subset of $\mathbb{R}$ or, alternatively, for some positive numbers $a$ and $b$, isometric with $a$ set $\{(0,0),(a, 0),(0, b),(a, b)\} \subset \mathbb{R}^{2}$ equipped with the metric $d_{1}$, where $d_{1}(x, y)=\left|x_{1}-y_{1}\right|+\left|x_{2}-y_{2}\right|$ for $x=\left(x_{1}, x_{2}\right), y=\left(y_{1}, y_{2}\right)$.

In fact, Menger proved in [9] that a metric space of more than $n+3$ points for which each of its subsets of $n+2$ points is isometric with a subset of $\mathbb{R}^{n}$, is isometric with a subset of $\mathbb{R}^{n}$. He also showed that for each $n$ there is a metric space of $n+3$ points for which each of its subsets of $n+2$ points is isometric with a subset of $\mathbb{R}^{n}$, but which is not isometric with a subset of $\mathbb{R}^{n}$. For the proof see also [1]. 
3. Metric spaces with the property $\Omega^{*}$. We will first show that a compact metric space with the property $\Omega^{*}$ is a Lipschitz image of a compact set of real numbers.

Lemma 3.1. Let $E$ be a metric space which has the complete property $\Omega^{*}$ with a constant $\alpha>0$, and let

$$
R=\frac{d(E) \sqrt{1+\sin \alpha}}{2(\sqrt{2}+\sqrt{1+\sin \alpha})} .
$$

Then for all $a \in E$ and $r<R$ there exist $A \subset[0,1]$ and a bijection $f: A \rightarrow$ $B(a, r)$ such that

$$
d(B(a, r))|s-t| \leq d(f(s), f(t)) \leq \frac{d(B(a, r))}{\sin \alpha}|s-t|
$$

for all $s, t \in A$.

Proof. Let $a \in E, r<R$ and $\varepsilon=\sin \alpha(>0)$. For every triple $\{x, y, z\} \subset E$ we have by the assumption $d(x, y)^{2} \geq d(x, z)^{2}+d(y, z)^{2}+2 \varepsilon d(x, z) d(y, z)$ whenever $d(x, y)=d(\{x, z, y\})$. Set

$$
d^{\prime}=\left(\frac{\sqrt{2}}{\sqrt{1+\varepsilon}}+1\right) r
$$

Since $d^{\prime}<d(E) / 2$, there exists $b \in E$ such that $d(a, b)>d^{\prime}$. Define a function

$$
g: B(a, r) \rightarrow[d(a, b)-r, d(a, b)+r]
$$

by setting $g(x)=d(x, b)$. Let $x, y \in B(a, r)$. Now

$$
\begin{aligned}
d(x, b)^{2}+d(y, b)^{2}+2 \varepsilon d(x, b) d(y, b) & \geq 2(d(a, b)-r)^{2}+2 \varepsilon(d(a, b)-r)^{2} \\
& >2(1+\varepsilon)\left(d^{\prime}-r\right)^{2}=4 r^{2} \geq d(x, y)^{2},
\end{aligned}
$$

and thus $d(x, y)<d(\{x, b, y\})$. Suppose $d(x, b) \geq d(y, b)$. Since

$$
d(x, b)^{2} \geq d(y, b)^{2}+d(x, y)^{2}+2 \varepsilon d(y, b) d(x, y) \geq(d(y, b)+\varepsilon d(x, y))^{2},
$$

we get

$$
\varepsilon d(x, y) \leq d(x, b)-d(y, b)=|g(x)-g(y)| \leq d(x, y)
$$

and further for $s, t \in g(B(a, r))$,

$$
|s-t| \leq d\left(g^{-1}(s), g^{-1}(t)\right) \leq \frac{1}{\varepsilon}|s-t|,
$$

where $g^{-1}: g(E) \rightarrow E$ is the inverse of $g$. If $B(a, r)$ contains at least two points, we take $A=h^{-1}(g(B(a, r))) \subset[0,1]$ and $f=g^{-1} \circ h: A \rightarrow B(a, r)$, where $h(s)=d(B(a, r)) s+\inf g(B(a, r))$.

Now we get immediately the following result. 
Proposition 3.2. Let $E$ be a compact metric space with the property $\Omega^{*}$. Then there exist $A \subset[0,1]$ and a Lipschitz surjection $f: A \rightarrow E$. (Moreover, $f$ and $A$ can be chosen such that $A=\bigcup_{i=1}^{n} A_{i}$, where $n \in \mathbb{N}$, the sets $A_{i}$ are compact and the restrictions $\left.f\right|_{A_{i}}$ are bi-Lipschitz maps.)

By the proof of Lemma 3.1 it is clear that we do not have to suppose so much in the previous proposition.

Proposition 3.3. Let $E$ be a compact metric space and suppose that for all $a \in E$ there are $r>0, \alpha>0$ and $b \in E \backslash\{a\}$ such that $\max \varangle\{b, c, d\} \geq$ $\pi / 2+\alpha$ for all $c, d \in B(a, r)$. Then there exist $A \subset[0,1]$ and a Lipschitz surjection $f: A \rightarrow E$.

Further we get the following corollaries.

COROLlary 3.4. Let $E$ be a compact metric space and suppose that for all $a \in E$ there is $r>0$ such that $c(x, y, z) d(x, y)<\sqrt{3}$ for $x, y, z \in B(a, r)$. Then there exist $A \subset[0,1]$ and a Lipschitz surjection $f: A \rightarrow E$.

Proof. By (1), the condition $c(x, y, z) d(x, y)<\sqrt{3}$ implies that $\sin \alpha<$ $\sqrt{3} / 2$, where $\alpha$ is the angle at $z$ for the triple $\{x, y, z\}$. So by the assumption, for every $a \in E$ there is $r>0$ such that $\max \varangle\{x, y, z\}>2 \pi / 3$ whenever $x, y, z \in B(a, r)$. Since $E$ has the property $\Omega^{*}$, the corollary follows from Proposition 3.2.

COROLlary 3.5. Let $E$ be a compact metric space and suppose that there is $M \in \mathbb{R}$ such that $c(x, y, z) \leq M$ for all $x, y, z \in E$. Then there exist $A \subset[0,1]$ and a Lipschitz surjection $f: A \rightarrow E$.

Now we are going to show that every bounded metric space with the complete property $\Omega^{*}$ is a Lipschitz image of a bounded set of real numbers. We also try to estimate the optimal Lipschitz constant. For that purpose we use the following lemma.

LEMma 3.6. Let $E$ be a bounded metric space which has the complete property $\Omega^{*}$ with a constant $\alpha>0$, and let

$$
R=\frac{d(E)}{\sqrt{2} \sqrt{1+\sin \alpha}}
$$

and $a \in E$. Then $d(x, y)<R$ for all $x, y \in E \backslash B(a, R)$.

Proof. Let $a \in E$ and $x, y \in E \backslash B(a, R)$. Suppose $d(x, y) \geq R$. Then

$$
\max \{d(x, a), d(y, a), d(x, y)\}>\sqrt{2} \sqrt{1+\sin \alpha} R=d(E),
$$

which is a contradiction.

THEOREM 3.7. If a bounded metric space $E$ has the complete property $\Omega^{*}$ with a constant $\alpha>0$, then there exist $A \subset[0,1]$ and a bijective map 
$f: A \rightarrow E$ such that

$$
d(E) \frac{\sin \alpha}{2}|s-t| \leq d(f(s), f(t)) \leq d(E) \frac{9}{2 \sin \alpha}|s-t|
$$

for all $s, t \in A$.

Proof. Let $a \in E, d=d(E), \varepsilon=\sin \alpha$ and

$$
\begin{aligned}
& \lambda=\max \left\{\frac{1}{\sqrt{1+4 \varepsilon+2 \varepsilon^{2}}}, \frac{\sqrt{2}}{\sqrt{3+\varepsilon+2 \sqrt{2} \varepsilon \sqrt{1+\varepsilon}}}\right\}, \\
& R=\frac{d}{\sqrt{2} \sqrt{1+\varepsilon}} .
\end{aligned}
$$

For $r>0$ define

$$
E_{r}=\{x \in E: \lambda r<d(x, a) \leq r\}, \quad \delta_{r}=\frac{\lambda r \sqrt{1+\varepsilon}}{\sqrt{2}} .
$$

Let $r>0$ and $x_{1}, x_{2}, x_{3} \in E_{r}$. We shall first show that one of the distances $d\left(x_{1}, x_{2}\right), d\left(x_{1}, x_{3}\right)$ and $d\left(x_{2}, x_{3}\right)$ must be less than $\delta_{r}$. Set $d_{i}=d\left(x_{i}, a\right)$ and $d_{i j}=d\left(x_{i}, x_{j}\right)$ for $i, j=1,2,3$, and suppose $d_{1} \leq d_{2} \leq d_{3}$. Then at least one in each of the following three pairs of inequalities is true:

$$
\begin{aligned}
d_{2}^{2} & \geq d_{1}^{2}+d_{12}^{2}+2 \varepsilon d_{1} d_{12}, \\
d_{12}^{2} & \geq d_{1}^{2}+d_{2}^{2}+2 \varepsilon d_{1} d_{2}, \\
d_{3}^{2} & \geq d_{1}^{2}+d_{13}^{2}+2 \varepsilon d_{1} d_{13}, \\
d_{13}^{2} & \geq d_{1}^{2}+d_{3}^{2}+2 \varepsilon d_{1} d_{3}, \\
d_{3}^{2} & \geq d_{2}^{2}+d_{23}^{2}+2 \varepsilon d_{2} d_{23}, \\
d_{23}^{2} & \geq d_{2}^{2}+d_{3}^{2}+2 \varepsilon d_{2} d_{3} .
\end{aligned}
$$

At least one of inequalities (12), (14) and (16) must be true. Otherwise, we would have (13), (15) and (17). In that case, the smallest distance in $\left\{x_{1}, x_{2}, x_{3}\right\}$ would be at least $\sqrt{d_{1}^{2}+d_{2}^{2}+2 \varepsilon d_{1} d_{2}}$ and another one at least $\sqrt{d_{1}^{2}+d_{3}^{2}+2 \varepsilon d_{1} d_{3}}$. The third distance is, of course, not more than $d_{2}+d_{3}$. Now we have

$$
\begin{aligned}
d_{1}^{2} & +d_{2}^{2}+2 \varepsilon d_{1} d_{2}+d_{1}^{2}+d_{3}^{2}+2 \varepsilon d_{1} d_{3} \\
& +2 \varepsilon \sqrt{d_{1}^{2}+d_{2}^{2}+2 \varepsilon d_{1} d_{2}} \sqrt{d_{1}^{2}+d_{3}^{2}+2 \varepsilon d_{1} d_{3}}-\left(d_{2}+d_{3}\right)^{2} \\
= & 2 d_{1}^{2}+2 \varepsilon d_{1} d_{2}+2 \varepsilon d_{1} d_{3}+2 \varepsilon \sqrt{d_{1}^{2}+d_{2}^{2}+2 \varepsilon d_{1} d_{2}} \sqrt{d_{1}^{2}+d_{3}^{2}+2 \varepsilon d_{1} d_{3}}-2 d_{2} d_{3} \\
> & 2\left((\lambda r)^{2}+2 \varepsilon(\lambda r)^{2}+2 \varepsilon(1+\varepsilon)(\lambda r)^{2}-r^{2}\right) \\
= & 2 r^{2}\left(\left(1+4 \varepsilon+2 \varepsilon^{2}\right) \lambda^{2}-1\right) \geq 0,
\end{aligned}
$$


and thus we would have $\max \varangle\left\{x_{1}, x_{2}, x_{3}\right\}<\pi / 2+\alpha$, which is a contradiction. If $x, y \in E_{r}$ are such that $d(x, y) \leq \max \{d(x, a), d(a, y)\}$, then

$$
d(x, y)<r\left(\sqrt{\left(\varepsilon^{2}-1\right) \lambda^{2}+1}-\varepsilon \lambda\right) \leq \frac{\lambda r \sqrt{1+\varepsilon}}{\sqrt{2}}
$$

by (3) and the choice of $\lambda$. Thus $\min \left\{d_{12}, d_{13}, d_{23}\right\}<\delta_{r}$. If $x, y \in E_{r}$ are such that $d(x, y)=d(\{x, a, y\})$, then

$$
\begin{aligned}
d(x, y) & \geq \sqrt{d(x, a)^{2}+d(y, a)^{2}+2 \varepsilon d(x, a) d(y, a)}>\sqrt{2(\lambda r)^{2}+2 \varepsilon(\lambda r)^{2}} \\
& =\lambda r \sqrt{2} \sqrt{1+\varepsilon} .
\end{aligned}
$$

This means that for all $r>0$ and $x, y \in E_{r}$ either $d(x, y)$ $>2 \delta_{r}$ or $d(x, y)<\delta_{r}$ and in the latter case $d(x, y)<\min \{d(x, a), d(y, a)\}$. Further $E_{r}$ has a unique decomposition into two sets $A_{r}$ and $B_{r}$ such that $d\left(A_{r}\right) \leq \delta_{r}, d\left(B_{r}\right) \leq \delta_{r}$ and $d\left(A_{r}, B_{r}\right) \geq 2 \delta_{r}$. (If $E_{r} \neq \emptyset$ we can choose $z \in E_{r}$ and take $A_{r}=\left\{x \in E_{r}: d(x, z)<\delta_{r}\right\}$ and $\left.B_{r}=E_{r} \backslash A_{r}.\right)$

Set $F_{-1}=E \backslash B(a, R)$ and $G_{-1}=\emptyset$. For all $k$ define sets $F_{k}$ and $G_{k}$ inductively as follows. Let $k \in \mathbb{N}$ and suppose that we have defined $F_{k-1}$ and $G_{k-1}$ such that $E_{\lambda^{k-1} R}=F_{k-1} \cup G_{k-1}$. Suppose first that there exists $\lambda^{k} R<r_{k}<\lambda^{k-1} R$ such that $E_{r_{k}} \cap E_{\lambda^{k-1} R} \neq \emptyset$ and $E_{r_{k}} \cap E_{\lambda^{k} R} \neq \emptyset$. Choose $z_{k-1} \in E_{r_{k}} \cap E_{\lambda^{k-1} R}$ and $w_{k} \in E_{r_{k}} \cap E_{\lambda^{k} R}$. Then we have the following alternatives:

$$
\begin{aligned}
& z_{k-1} \in F_{k-1} \quad \text { and } \quad d\left(z_{k-1}, w_{k}\right)<\delta_{r_{k}} \text {, } \\
& z_{k-1} \in F_{k-1} \quad \text { and } \quad d\left(z_{k-1}, w_{k}\right)>2 \delta_{r_{k}} \text {, } \\
& z_{k-1} \in G_{k-1} \quad \text { and } \quad d\left(z_{k-1}, w_{k}\right)<\delta_{r_{k}} \text {, } \\
& z_{k-1} \in G_{k-1} \quad \text { and } \quad d\left(z_{k-1}, w_{k}\right)>2 \delta_{r_{k}} .
\end{aligned}
$$

If (18) or (21) is true, we set $F_{k}=\left\{x \in E_{\lambda^{k} R}: d\left(x, w_{k}\right)<\delta_{\lambda^{k} R}\right\}$ and $G_{k}=E_{\lambda^{k} R} \backslash F_{k}$. Otherwise we put $G_{k}=\left\{x \in E_{\lambda^{k} R}: d\left(x, w_{k}\right)<\delta_{\lambda^{k} R}\right\}$ and $F_{k}=E_{\lambda^{k} R} \backslash G_{k}$. If $E_{r} \cap E_{\lambda^{k-1} R}=\emptyset$ or $E_{r} \cap E_{\lambda^{k} R}=\emptyset$ for all $\lambda^{k} R<r<\lambda^{k-1} R$, we define $F_{k}$ and $G_{k}$ arbitrarily so that $E_{\lambda^{k} R}=F_{k} \cup G_{k}, d\left(F_{k}\right) \leq \delta_{\lambda^{k} R}$, $d\left(G_{k}\right) \leq \delta_{\lambda^{k} R}$ and $d\left(F_{k}, G_{k}\right) \geq 2 \delta_{\lambda^{k} R}$.

Set $F=\bigcup_{k=-1}^{\infty} F_{k}$ and $G=\bigcup_{k=0}^{\infty} G_{k}$. Then $E=F \cup G \cup\{a\}$. Define a function $g: E \rightarrow[-R, d]$ by setting

$$
g(x)= \begin{cases}-d(x, a) & \text { for } x \in G, \\ d(x, a) & \text { for } x \in F \cup\{a\} .\end{cases}
$$

We now show that $g$ is bi-Lipschitz.

If $x, y \in F_{k}$ or $x, y \in G_{k}$ for some $k \in \mathbb{N}$, we have, by (3),

$$
\varepsilon d(x, y) \leq|d(x, a)-d(y, a)|=|g(x)-g(y)| \leq d(x, y),
$$


because $d(x, y) \leq \delta_{\lambda^{k} R}$ implies $d(x, y)<d(\{x, a, y\})$. The same is true for $x, y \in F_{-1}$ by Lemma 3.6. If $x \in F_{k}$ and $y \in G_{k}$ for some $k \in \mathbb{N}$, we have

$$
1 \leq \frac{d(x, a)+d(y, a)}{d(x, y)}=\frac{|g(x)-g(y)|}{d(x, y)} \leq \frac{2 \lambda^{k} R}{2 \delta_{\lambda^{k} R}}=\frac{\sqrt{2}}{\lambda \sqrt{1+\varepsilon}} .
$$

From now on we suppose that $x, y \in E$ are such that $d(y, a) \leq d(x, a)$ and $x \neq a$.

$$
\begin{aligned}
& \text { If } d(y, a) \leq \lambda d(x, a) \text {, then } \\
& \frac{1-\lambda}{1+\lambda} \leq \frac{d(x, a)-d(y, a)}{d(x, a)+d(y, a)} \leq \frac{|g(x)-g(y)|}{d(x, y)} \leq \frac{d(x, a)+d(y, a)}{d(x, a)-d(y, a)} \leq \frac{1+\lambda}{1-\lambda} .
\end{aligned}
$$

Suppose $d(y, a)>\lambda d(x, a)$. Then either $x, y \in F_{-1}$ or $x, y \in E_{\lambda^{k-1} R} \cup E_{\lambda^{k} R}$ for some $k \in \mathbb{N}$. We have to check the case $x \in E_{\lambda^{k-1} R}$ and $y \in E_{\lambda^{k} R}$ for some $k$. Since $d(y, a) \leq d(x, a) \leq d(y, a) / \lambda$, we have $x \in E_{r_{k}}$ or $y \in E_{r_{k}}$. We may assume that $x \in E_{r_{k}}$. Then $x, y, w_{k} \in E_{d(x, a)}$.

Suppose first that $d\left(x, z_{k-1}\right)<\delta_{\lambda^{k-1} R}, d\left(y, w_{k}\right)<\delta_{\lambda^{k} R}$ and $d\left(z_{k-1}, w_{k}\right)$ $<\delta_{r_{k}}$. Then we have either $x \in F_{k-1}, y \in F_{k}$ and (18), or $x \in G_{k-1}, y \in G_{k}$ and (20). Since $\delta_{\lambda^{k-1} R}<2 \delta_{r_{k}}$, we have $d\left(x, z_{k-1}\right)<\delta_{r_{k}}$. Thus $d\left(x, w_{k}\right) \leq$ $d\left(x, z_{k-1}\right)+d\left(z_{k-1}, w_{k}\right)<2 \delta_{r_{k}}$ and so $d\left(x, w_{k}\right)<\delta_{r_{k}}$, because $x, w_{k} \in E_{r_{k}}$. Since $\delta_{r_{k}}<2 \delta_{d(x, a)}$ and $x, w_{k} \in E_{d(x, a)}$, we further have $d\left(x, w_{k}\right)<\delta_{d(x, a)}$. Therefore $d(x, y) \leq d\left(x, w_{k}\right)+d\left(y, w_{k}\right)<\delta_{d(x, a)}+\delta_{\lambda^{k} R}<2 \delta_{d(x, a)}$. Since $x, y \in E_{d(x, a)}$, we have $d(x, y)<\delta_{d(x, a)}$ and $(22)$.

Suppose now that $d\left(x, z_{k-1}\right)<\delta_{\lambda^{k-1} R}, d\left(y, w_{k}\right)<\delta_{\lambda^{k} R}$ and $d\left(z_{k-1}, w_{k}\right)$ $>2 \delta_{r_{k}}$. Then we have either $x \in F_{k-1}, y \in G_{k}$ and (19), or $x \in G_{k-1}$, $y \in F_{k}$ and (21). Since $\delta_{\lambda^{k-1} R}<2 \delta_{r_{k}}$, we have $d\left(x, z_{k-1}\right)<\delta_{r_{k}}$. Thus $d\left(x, w_{k}\right) \geq d\left(w_{k}, z_{k-1}\right)-d\left(x, z_{k-1}\right)>\delta_{r_{k}}$ and so $d\left(x, w_{k}\right)>2 \delta_{r_{k}}$, because $x, w_{k} \in E_{r_{k}}$. Therefore $d(x, y) \geq d\left(x, w_{k}\right)-d\left(y, w_{k}\right)>2 \delta_{r_{k}}-\delta_{\lambda^{k} R}>\delta_{d(x, a)}$. Since $x, y \in E_{d(x, a)}$, we have $d(x, y)>2 \delta_{d(x, a)}$ and

$$
1 \leq \frac{d(x, a)+d(y, a)}{d(x, y)}=\frac{|g(x)-g(y)|}{d(x, y)} \leq \frac{2 d(x, a)}{2 \delta_{d(x, a)}}=\frac{\sqrt{2}}{\lambda \sqrt{1+\varepsilon}} .
$$

The other cases can be treated similarly. The inequality

$$
\frac{1-\lambda}{1+\lambda} \leq \frac{|g(x)-g(y)|}{d(x, y)} \leq \frac{1+\lambda}{1-\lambda}
$$

holds for all $x, y \in E$. Thus we get a set $A \subset[0,1]$ and a surjection $f: A \rightarrow E$ such that

$$
\begin{aligned}
d(E) \frac{1+\sqrt{2} \sqrt{1+\varepsilon}}{\sqrt{2} \sqrt{1+\varepsilon}} \frac{1-\lambda}{1+\lambda}|s-t| & \leq d(f(s), f(t)) \\
& \leq d(E) \frac{1+\sqrt{2} \sqrt{1+\varepsilon}}{\sqrt{2} \sqrt{1+\varepsilon}} \frac{1+\lambda}{1-\lambda}|s-t|
\end{aligned}
$$


and further the estimate

$$
d(E) \frac{\varepsilon}{2}|s-t| \leq d(f(s), f(t)) \leq d(E) \frac{9}{2 \varepsilon}|s-t|
$$

for all $s, t \in A$.

We now give an example of a compact and connected metric space which has an order and the complete property $\Omega$, but which is not a Lipschitz image of a bounded set of real numbers.

ExAmPLE 3.8. Let $1<p<2$ and $x \in \ell^{p} \backslash \ell^{1}, x=\left(x_{k}\right)_{k=1}^{\infty}$, where $x_{k} \geq 0$ for every $k$. Set

$$
E=\left\{\sum_{k=1}^{n} x_{k} e_{k}+t e_{n+1}: n \in \mathbb{N}, t \in\left[0, x_{n+1}\right]\right\} \cup\{x\} \subset \ell^{p},
$$

where $\left\{e_{k}: k \in \mathbb{N}\right\}$ is the standard basis of $\ell^{p}$. Now $j: y \mapsto\|y\|_{p}$ is an order on $E$ and maps $E$ onto $\left[x_{1},\|x\|_{p}\right]$. We check that every triple in $E$ contains an obtuse angle. Let

$$
a_{1}=\sum_{k=1}^{n_{1}} x_{k} e_{k}, \quad a_{2}=\sum_{k=1}^{n_{2}} x_{k} e_{k}+t e_{n_{2}+1}, \quad a_{3}=\sum_{k=1}^{n_{3}} x_{k} e_{k}
$$

where $\left\|a_{1}\right\|_{p}<\left\|a_{2}\right\|_{p}<\left\|a_{3}\right\|_{p}, n_{1} \leq n_{2}<n_{3} \leq \infty$, and $t \in\left[0, x_{n_{2}+1}\right]$. Set

$$
A=\sum_{k=n_{1}+1}^{n_{2}} x_{k}^{p}, \quad B=\sum_{k=n_{2}+2}^{n_{3}} x_{k}^{p} \text {. }
$$

Now

$$
\begin{aligned}
d\left(a_{1}, a_{3}\right)^{2}- & d\left(a_{1}, a_{2}\right)^{2}-d\left(a_{2}, a_{3}\right)^{2}=\left\|a_{1}-a_{3}\right\|_{p}^{2}-\left\|a_{1}-a_{2}\right\|_{p}^{2}-\left\|a_{2}-a_{3}\right\|_{p}^{2} \\
& =\left(A+x_{n_{2}+1}^{p}+B\right)^{2 / p}-\left(A+t^{p}\right)^{2 / p}-\left(\left(x_{n_{2}+1}-t\right)^{p}+B\right)^{2 / p} \\
\geq & \left(A+x_{n_{2}+1}^{p}+B\right)^{2 / p}-\left(A+t^{p}\right)^{2 / p}-\left(x_{n_{2}+1}^{p}-t^{p}+B\right)^{2 / p}>0
\end{aligned}
$$

because $(a+b)^{s}>a^{s}+b^{s}$ for $a, b>0$ and $s>1$. So for $\left\{a_{1}, a_{2}, a_{3}\right\}$ the angle at $a_{2}$ is obtuse. However, even

$$
E^{\prime}=\left\{\sum_{k=1}^{n} x_{k} e_{k}: n \in \mathbb{N}\right\} \subset E
$$

cannot be a Lipschitz image of a bounded set of real numbers when $x \notin \ell^{1}$. Namely, if $A \subset[0,1]$ and $f: A \rightarrow E^{\prime}$ is a Lipschitz map such that

$$
\left\{\sum_{k=1}^{n} x_{k} e_{k}: n=1, \ldots, n_{0}\right\} \subset f(A),
$$

the Lipschitz constant of $f$ must be at least $\sum_{k=2}^{n_{0}} x_{k}$. 
4. Connected, ordered and ptolemaic spaces. Let $\mathcal{M}$ be the class of all bounded metric spaces. For $E \in \mathcal{M}$ we define

$$
l(E)=\inf \{\operatorname{Lip}(f): f: A \rightarrow E \text { is a surjection and } A \subset[0,1]\},
$$

where $\operatorname{Lip}(f) \in[0, \infty]$ is the Lipschitz constant of $f$. For $\mathcal{A} \subset \mathcal{M}$ we set

$$
\widetilde{L}(\mathcal{A})=\sup \{l(E) / d(E): E \in \mathcal{A}\} .
$$

Further for $0<\varepsilon \leq 1$ and $\mathcal{A} \subset \mathcal{M}$ we put $L(\varepsilon, \mathcal{A})=\widetilde{L}(\Omega(\varepsilon) \cap \mathcal{A})$. Clearly $\varepsilon \mapsto L(\varepsilon, \mathcal{A})$ is a decreasing function on $] 0,1]$ for fixed $\mathcal{A} \subset \mathcal{M}$, and $L(\varepsilon, \mathcal{A}) \leq$ $L(\varepsilon, \mathcal{B})$ if $\mathcal{A} \subset \mathcal{B} \subset \mathcal{M}$. By Proposition 2.4 we have $L(1, \mathcal{M})=3 / 2$, and $L(\varepsilon, \mathcal{M}) \leq C / \varepsilon$ with some constant $C>0$ by Theorem 3.7.

We denote by $\mathcal{C}$ the class of connected metric spaces, and by $\mathcal{O}$ the class of metric spaces which have an order. We next show that $L(\varepsilon, \mathcal{C})=$ $L(\varepsilon, \mathcal{O})=1 / \varepsilon$ for $0<\varepsilon \leq 1$.

Lemma 4.1. Let $E$ be a connected metric space such that $\max \varangle\{x, y, z\}$ $\geq \pi / 2$ for every triple $\{x, y, z\} \subset E$. If $f: E \rightarrow \mathbb{R}$ is a homeomorphism onto its image, then $f$ is an order.

Proof. We can of course assume that $E$ contains more than one point. Suppose that $f$ is not an order. Then there exists $\{x, y, z\} \subset E$ such that

$$
f(x)<f(y)<f(z)
$$

and $d(x, z) \leq \max \{d(x, y), d(y, z)\}$. We can assume $x z y$, as $\max \varangle\{x, y, z\}$ $\geq \pi / 2$. Define $g: f(E) \rightarrow \mathbb{R}$ by setting $g(a)=d\left(x, f^{-1}(a)\right)$, where $f^{-1}$ is the inverse of $f$. Now $g$ is continuous. Since $f(E)$ is connected, $[f(x), f(y)] \subset$ $f(E)$. Let $b \in[f(x), f(y)]$ be such that $g(b)=\max \{g(a): a \in[f(x), f(y)]\}$. Now $b \in] f(x), f(y)[$ because of (23). Now

$$
d\left(x, f^{-1}(c)\right)=g(c) \uparrow g(b)=d\left(x, f^{-1}(b)\right)
$$

as $c \uparrow b$ and

$$
d\left(x, f^{-1}(e)\right)=g(e) \uparrow g(b)=d\left(x, f^{-1}(b)\right)
$$

as $e \downarrow b$, where $d\left(x, f^{-1}(b)\right)>0$. Further by the continuity of $f^{-1}$ we simultaneously have $d\left(f^{-1}(c), f^{-1}(e)\right) \downarrow 0$. From this we conclude that $E$ contains a triple whose maximum angle is less than $\pi / 2$, which is a contradiction.

Proposition 4.2. $L(\varepsilon, \mathcal{C})=L(\varepsilon, \mathcal{O})=1 / \varepsilon$ for all $\varepsilon \in] 0,1]$.

Proof. Let $0<\varepsilon \leq 1$. By Theorem 3.7 and Lemma 4.1 we have $L(\varepsilon, \mathcal{C})$ $\leq L(\varepsilon, \mathcal{O})$. Clearly $L(\varepsilon, \mathcal{O}) \leq 1 / \varepsilon$. Namely, if $E \in \Omega(\varepsilon) \cap \mathcal{O}$, it follows from Theorem 3.7 that the completion of $E$ is compact. Since clearly also the completion of $E$ is in $\Omega(\varepsilon) \cap \mathcal{O}$, we may assume that $E$ is compact. Take $a, b \in E$ such that $d(a, b)=d(E)$ and define $g: E \rightarrow[0, d(E)]$ by setting $g(x)=d(x, a)$. As before, we see by (3) that the inverse of $g$ is $1 / \varepsilon$-Lipschitz from $d(E)$ to $E$. 
We are left to show $L(\varepsilon, \mathcal{C}) \geq 1 / \varepsilon$. We define a metric $d$ on an interval $[0, N], N \in \mathbb{N}$, as follows: Define real numbers $r_{k}, k=0,1, \ldots$, by setting $r_{0}=0$ and

$$
r_{k+1}=\sqrt{r_{k}^{2}+1+2 \varepsilon r_{k}}
$$

Let $x, y \in[0, N]$ with $x<y$. If $\mathbb{N} \cap[x, y]=\emptyset$, we set $d(x, y)=|x-y|$. Else we put $m=\inf (\mathbb{N} \cap[x, y]), M=\sup (\mathbb{N} \cap[x, y]), s=\min \{y-M, m-x\}$ and $t=\max \{y-M, m-x\}$. Then we set

$$
d(x, y)=\sqrt{u^{2}+s^{2}+2 \varepsilon u s},
$$

where

$$
u=\sqrt{r_{M-m}^{2}+t^{2}+2 \varepsilon r_{M-m} t} .
$$

Denote this metric space by $E_{N}$. Then $E_{N} \in \Omega(\varepsilon) \cap \mathcal{C}$. Since $r_{k+1}-r_{k} \rightarrow \varepsilon$ as $k \rightarrow \infty$, we have

$$
\frac{l\left(E_{N}\right)}{d\left(E_{N}\right)}=\frac{N}{r_{N}} \rightarrow \frac{1}{\varepsilon}
$$

as $N \rightarrow \infty$.

We say that a metric space $E$ has the four-point property if any subset of four points of $E$ is isometric with some subset of $\mathbb{R}^{3}$. $E$ is called ptolemaic provided for all $x, y, z, w \in E$ the inequality $d(x, y) d(z, w)+d(x, z) d(y, w) \geq$ $d(x, w) d(y, z)$ is true. Denote the class of metric spaces with the four-point property by $\mathcal{F}$, and the class of ptolemaic metric spaces by $\mathcal{P}$. Since $\mathbb{R}^{3}$ is ptolemaic, we have $\mathcal{F} \subset \mathcal{P}$. It is easy to construct metric spaces which are ptolemaic but which do not have the four-point property. For example we can take a quadruple such that one distance between points equals 2 while the other five distances are 1 . Since $\mathcal{F} \cap \Omega(\sqrt{3} / 2) \subset \mathcal{O}$, we have at least $L(\varepsilon, \mathcal{F}) \leq 1 / \varepsilon$ for $\sqrt{3} / 2 \leq \varepsilon \leq 1$. We now show that $L(\varepsilon, \mathcal{P}) \downarrow 1$ as $\varepsilon \uparrow 1$.

Lemma 4.3. Let $E$ be a ptolemaic metric space with the complete property $\Omega$. Then $\min \{d(x, y), d(z, w)\}<\max \{d(x, z), d(x, w), d(y, z), d(y, w)\}$ for any four pairwise distinct points $x, y, z, w \in E$.

Proof. Otherwise

$$
\begin{aligned}
d(x, y)^{2} d(z, w)^{2} & >\frac{\left(d(x, z)^{2}+d(y, w)^{2}+d(x, w)^{2}+d(y, z)^{2}\right)^{2}}{4} \\
& \geq \frac{(2 d(x, z) d(y, w)+2 d(x, w) d(y, z))^{2}}{4} \\
& =(d(x, z) d(y, w)+d(x, w) d(y, z))^{2},
\end{aligned}
$$

which means that $E$ is not ptolemaic.

Proposition 4.4. $L(\varepsilon, \mathcal{P}) \downarrow 1$ as $\varepsilon \uparrow 1$. 
Proof. Let $E \in \Omega(\varepsilon) \cap \mathcal{P}$. It follows from Theorem 3.7 that the completion of $E$ is compact. Since clearly also the completion of $E$ is in $\Omega(\varepsilon) \cap \mathcal{P}$, we can assume that $E$ is compact. Let $a, b \in E$ be such that $d(a, b)=d(E)$. We define $g: E \rightarrow[0, d(E)]$ by setting $g(x)=d(x, a)$. Let $x, y \in E \backslash\{a, b\}$. If $d(x, y) \leq \max \{d(x, a), d(a, y)\}$, then $|g(x)-g(y)| \geq \varepsilon d(x, y)$ by (3). Suppose $x a y$. By the previous lemma, $d(x, y) \leq \max \{d(x, b), d(b, y)\}$. We may assume that $d(y, b)>d(x, b)$. Set $d=d(E), p=d(x, a), q=d(y, a), r=d(x, y)$, $s=d(y, b)$ and $t=d(x, b)$. Then $d^{2} \geq q^{2}+s^{2}+2 \varepsilon q s$, which gives

$$
s \leq \sqrt{d^{2}+\left(\varepsilon^{2}-1\right) q^{2}}-\varepsilon q .
$$

We also have $t \geq d-p$ and $r^{2} \geq q^{2}+p^{2}+2 \varepsilon q p$. Thus we get

$$
\begin{aligned}
& s^{2}-t^{2}-r^{2} \\
& \leq d^{2}+\left(2 \varepsilon^{2}-1\right) q^{2}-2 \varepsilon q \sqrt{d^{2}+\left(\varepsilon^{2}-1\right) q^{2}}-d^{2}-p^{2}+2 d p-q^{2}-p^{2}-2 \varepsilon q p \\
& =2\left[\left(\varepsilon^{2}-1\right) q^{2}-p^{2}+d p-\varepsilon q p-\varepsilon q \sqrt{d^{2}+\left(\varepsilon^{2}-1\right) q^{2}}\right] \leq 2 p(d-p)
\end{aligned}
$$

and further

$$
\varepsilon \leq \frac{s^{2}-t^{2}-r^{2}}{2 r t} \leq \frac{p}{\sqrt{q^{2}+p^{2}+2 \varepsilon q p}} .
$$

This yields $p \geq \varepsilon(\varepsilon q+p)$, which gives $q \leq p(1-\varepsilon) / \varepsilon^{2}$. Thus

$$
\frac{|g(x)-g(y)|}{d(x, y)}=\frac{p-q}{r} \geq \frac{p-q}{p+q} \geq \frac{\varepsilon^{2}+\varepsilon-1}{\varepsilon^{2}-\varepsilon+1},
$$

and we get

$$
L(\varepsilon, \mathcal{P}) \leq \max \left\{\frac{\varepsilon^{2}-\varepsilon+1}{\varepsilon^{2}+\varepsilon-1}, \frac{1}{\varepsilon}\right\}=\frac{\varepsilon^{2}-\varepsilon+1}{\varepsilon^{2}+\varepsilon-1}
$$

when $(\sqrt{5}-1) / 2<\varepsilon \leq 1$. Therefore $L(\varepsilon, \mathcal{P}) \downarrow 1$ as $\varepsilon \uparrow 1$.

5. Travelling salesman theorem. Let $E$ be a bounded metric space and let $C_{1} \geq C_{2}>960$. For any $x \in E$ and $t>0$ we set

$$
\beta(x, t)=\sup \left\{c\left(z_{1}, z_{2}, z_{3}\right): z_{1}, z_{2}, z_{3} \in B(x, t), d\left(z_{i}, z_{j}\right) \geq C_{1}^{-1} t \forall i \neq j\right\} .
$$

We say that an increasing sequence $\left(\Delta_{k}\right)_{k \in \mathbb{Z}}$ of subsets of $E$ is a net of $E$ if for all $k \in \mathbb{Z}$,

(i) for any distinct $x, y \in \Delta_{k}, d(x, y)>2^{-k}$,

(ii) for any $x \in E$ there exists $y \in \Delta_{k}$ such that $d(x, y) \leq 2^{-k}$.

Now we define

$$
\beta(E)=\inf \left\{\sum_{k \in \mathbb{Z}} \sum_{x \in \Delta_{k}} \beta\left(x, C_{2} 2^{-k}\right)^{2}\left(2^{-k}\right)^{3}:\left(\Delta_{k}\right)_{k} \text { is a net of } E\right\} .
$$

For a bounded set $F \subset \mathbb{R}^{n}$ the conditions $\beta(F)<\infty$ and (4) are equivalent. We now sketch a proof for this. Let $F$ be a bounded set in $\mathbb{R}^{n}$. First, 
assume that $\beta(F)<\infty$. Then, by Theorem 5.3 below, we find $A \subset[0,1]$ and a Lipschitz surjection $f: A \rightarrow F$ such that $\operatorname{Lip}(f) \leq C(\beta(F)+d(F))$, where $C>0$ is an absolute constant. By the Kirszbraun theorem $f$ has an extension $\widetilde{f}:[0,1] \rightarrow \mathbb{R}^{n}$ such that $\operatorname{Lip}(\widetilde{f})=\operatorname{Lip}(f)$. Thus the result of Okikiolu (see [11]) gives

$$
\sum_{Q \in \mathcal{D}} \beta_{F}(Q)^{2} d(Q) \leq A_{1} \operatorname{Lip}(\tilde{f}) \leq A_{1} C(\beta(F)+d(F)),
$$

where $A_{1}$ is a constant depending on $n$ and $\mathcal{D}$ is the set of all dyadic cubes in $\mathbb{R}^{n}$.

If $Q \subset \mathbb{R}^{n}$ is a cube and $\lambda>1$, we define

$$
\beta_{F}^{\lambda}(Q)=\inf _{L} d(Q)^{-1} \sup \{d(y, L): y \in F \cap \lambda Q\},
$$

where the infimum is taken over all lines in $\mathbb{R}^{n}$ and $\lambda Q$ is the cube with the same center as $Q$ and sides parallel to the sides of $Q$, but whose diameter is $\lambda d(Q)$. It is not difficult to show that there exists a constant $A_{2}=A_{2}(n, \lambda)$ such that

$$
A_{2}^{-1} \sum_{Q \in \mathcal{D}} \beta_{F}^{\lambda}(Q)^{2} d(Q) \leq \int_{\mathbb{R}^{n}} \int_{0}^{\infty} \beta_{\infty}(x, t, F)^{2} \frac{d t}{t^{n}} d \mathcal{L}^{n} x \leq A_{2} \sum_{Q \in \mathcal{D}} \beta_{F}^{\lambda}(Q)^{2} d(Q),
$$

where $\mathcal{L}^{n}$ is the Lebesgue measure on $\mathbb{R}^{n}$ and

$$
\beta_{\infty}(x, t, F)=\inf _{L} t^{-1} \sup \{d(y, L): y \in F \cap B(x, t)\},
$$

where the infimum is taken over all lines in $\mathbb{R}^{n}$. In particular, for all $\lambda_{1}, \lambda_{2}$ $>1$ there exists a constant $A_{3}=A_{3}\left(n, \lambda_{1}, \lambda_{2}\right)$ such that

$$
\sum_{Q \in \mathcal{D}} \beta_{F}^{\lambda_{1}}(Q)^{2} d(Q) \leq A_{3} \sum_{Q \in \mathcal{D}} \beta_{F}^{\lambda_{2}}(Q)^{2} d(Q) .
$$

For $k \in \mathbb{Z}$ let $\mathcal{D}_{k}$ be the set of all dyadic cubes in $\mathbb{R}^{n}$ of side length $2^{-k}$. Choose $A_{4} \in \mathbb{Z}$ such that $(2 \log 2) A_{4} \geq \log n$ and define $\lambda=C_{2} 2^{A_{4}+1}+1$. Let $\left(\Delta_{k}\right)_{k}$ be a net of $F$ and fix $k \in \mathbb{Z}$. Then $\#\left(Q \cap \Delta_{k}\right) \leq 1$ for any $Q \in \mathcal{D}_{k+A_{4}}$. Fix $x \in \Delta_{k}$ and let $Q \in \mathcal{D}_{k+A_{4}}$ be such that $x \in Q$. Further let $z_{1}, z_{2}, z_{3} \in B\left(x, C_{2} 2^{-k}\right)$ be such that $d\left(z_{i}, z_{j}\right) \geq C_{1}^{-1} C_{2} 2^{-k}$ for all $i \neq j$. Since $B\left(x, C_{2} 2^{-k}\right) \subset \lambda Q$, by using (1) and some plane geometry we get

$$
\begin{aligned}
c\left(z_{1}, z_{2}, z_{3}\right)^{2}\left(2^{-k}\right)^{3} & =\frac{4 d\left(z_{1}, L\right)^{2}\left(2^{-k}\right)^{3}}{d\left(z_{1}, z_{2}\right)^{2} d\left(z_{1}, z_{3}\right)^{2}} \leq 4 C_{1}^{4} C_{2}^{-4} d\left(z_{1}, L\right)^{2} 2^{k} \\
& \leq \frac{4 C_{1}^{4} \sqrt{n} d\left(z_{1}, L\right)^{2}}{C_{2}^{4} 2^{A_{4}} d(Q)} \leq A_{5} \beta_{F}^{\lambda}(Q) d(Q),
\end{aligned}
$$

where $L$ is the line passing through $z_{2}$ and $z_{3}$ and $A_{5}=A_{5}\left(n, C_{1}, C_{2}\right)$ is a constant. Using (24), for some constant $A_{6}$ depending on $n, C_{1}$ and $C_{2}$ we 
get

$$
\begin{aligned}
\beta(F) & \leq \sum_{k \in \mathbb{Z}} \sum_{x \in \Delta_{k}} \beta\left(x, C_{2} 2^{-k}\right)^{2}\left(2^{-k}\right)^{3} \leq A_{5} \sum_{k \in \mathbb{Z}} \sum_{Q \in \mathcal{D}_{k+A_{3}}} \beta_{F}^{\lambda}(Q)^{2} d(Q) \\
& \leq A_{6} \sum_{Q \in \mathcal{D}} \beta_{F}(Q)^{2} d(Q) .
\end{aligned}
$$

Now we are going to show that for any bounded metric space $E$ the condition $\beta(E)<\infty$ implies that $E$ is a Lipschitz image of a bounded set of real numbers.

Lemma 5.1. Let $x, y, z \in \mathbb{R}^{2}$ be distinct points, $L_{y z}$ the line passing through $y$ and $z$, and $P: \mathbb{R}^{2} \rightarrow L_{y z}$ the orthogonal projection to $L_{y z}$. Set $d_{y x}=|y-x|, d_{x z}=|x-z|$ and $d_{y z}=|y-z|$. If $d_{y z}=|y-P(x)|+|P(x)-z|$, then

$$
\frac{c(x, y, z)^{2}}{8} \leq \frac{d_{y x}+d_{x z}-d_{y z}}{d_{y x} d_{x z}\left(d_{y x}+d_{x z}\right)} \leq \frac{c(x, y, z)^{2}}{4} .
$$

Proof. Define $s=|y-P(x)|, t=|P(x)-z|$ and $h=|x-P(x)|$. By the Pythagorean theorem

$$
\begin{aligned}
d_{y x}+d_{x z}-d_{y z} & =d_{y x}-s+d_{x z}-t=\frac{d_{y x}^{2}-s^{2}}{d_{y x}+s}+\frac{d_{x z}^{2}-t^{2}}{d_{x z}+t} \\
& =h^{2}\left(\frac{1}{d_{y x}+s}+\frac{1}{d_{x z}+t}\right)
\end{aligned}
$$

and by (1),

$$
c(x, y, z)=\frac{2 h}{d_{y x} d_{x z}} .
$$

Hence

$$
d_{y x}+d_{x z}-d_{y z}=\frac{c(x, y, z)^{2} d_{y x}^{2} d_{x z}^{2}}{4}\left(\frac{1}{d_{y x}+s}+\frac{1}{d_{x z}+t}\right),
$$

from which we get the conclusion.

Lemma 5.2. Let $E$ be a bounded metric space. Suppose that there exist $L<\infty$ and a dense subset $D \subset E$ such that there exist $U \subset[0,1]$ and an $L$-Lipschitz surjection $g: U \rightarrow F$ for every finite $F \subset D$. Then there exist $A \subset[0,1]$ and an L-Lipschitz surjection $f: A \rightarrow E$.

Proof. We can assume that $D$ is countable. Let $D=\left\{x_{1}, x_{2}, x_{3}, \ldots\right\}$ and define $D_{n}=\left\{x_{1}, \ldots, x_{n}\right\}$ and $N_{n}^{1}=\mathbb{N}_{n}=\{1, \ldots, n\}$ for $n \in \mathbb{N}$. By the hypothesis we have for every $n$ a permutation $\sigma_{n}^{1}$ of $\mathbb{N}_{n}$ such that $\sum_{i=1}^{n-1} d\left(x_{\sigma_{n}^{1}(i)}, x_{\sigma_{n}^{1}(i+1)}\right) \leq L$. Since for any $n$ there are only a finite number of permutations of $\mathbb{N}_{n}$, we can inductively choose sequences $\left(\sigma_{n}^{m}: N_{n}^{m} \rightarrow\right.$ $\left.N_{n}^{m}\right)_{n}$ of permutations such that for every $m \in \mathbb{N}$ the sequence $\left(\sigma_{n}^{m+1}\right)_{n}$ is 
a subsequence of $\left(\sigma_{n}^{m}\right)_{n}, \mathbb{N}_{m} \subset N_{1}^{m}$ and for every $n \in \mathbb{N}$ and $i, j \in \mathbb{N}_{m}$ the condition $\left(\sigma_{1}^{m}\right)^{-1}(i)<\left(\sigma_{1}^{m}\right)^{-1}(j)$ implies $\left(\sigma_{n}^{m}\right)^{-1}(i)<\left(\sigma_{n}^{m}\right)^{-1}(j)$. For each $n$ let $\sigma_{n}$ be the permutation of $\mathbb{N}_{n}$ such that for $i, j \in \mathbb{N}_{n}$ the condition $\left(\sigma_{n}\right)^{-1}(i)<\left(\sigma_{n}\right)^{-1}(j)$ implies $\left(\sigma_{n}^{n}\right)^{-1}(i)<\left(\sigma_{n}^{n}\right)^{-1}(j)$.

For every $n$ set $A_{n}=\left\{a_{1}^{n}, \ldots, a_{n}^{n}\right\}$, where $a_{k}^{n}=\sum_{i=1}^{k-1} d\left(x_{\sigma_{n}(i)}, x_{\sigma_{n}(i+1)}\right)$ for $k=1, \ldots, n$. Define a 1-Lipschitz bijection $f_{n}: A_{n} \rightarrow D_{n}$ by setting $f_{n}\left(a_{k}^{n}\right)=x_{\sigma_{n}(k)}$ for $k=1, \ldots, n$. Note that $A_{n} \subset[0, L]$ for every $n$ and the sequence $\left(a_{n}^{n}\right)$ is increasing. Now there exists a compact $A \subset[0, L]$ such that $A_{n} \rightarrow A$ in the Kuratowski sense:

(i) If $a=\lim _{n \rightarrow \infty} a_{n}^{m}$ for some subsequence $\left(a_{n}^{m}\right)$ of a sequence $\left(a_{n}\right)$ such that $a_{n} \in A_{n}$ for any $n$, then $a \in A$.

(ii) If $a \in A$, then there exists a sequence $\left(a_{n}\right)$ such that $a_{n} \in A_{n}$ for any $n$ and $a=\lim _{n \rightarrow \infty} a_{n}$.

Let $a \in A$ and let $\left(a_{n}\right)$ be a sequence such that $a_{n} \in A_{n}$ for any $n$ and $a_{n} \rightarrow a$ as $n \rightarrow \infty$. Let $m \geq n \geq 1$. Then there is $b \in A_{m}$ such that $f_{m}(b)=f_{n}\left(a_{n}\right)$ and $\left|a_{n}-b\right| \leq a_{m}^{m}-a_{n}^{n}$. Using this we get

$$
\begin{aligned}
d\left(f_{m}\left(a_{m}\right), f_{n}\left(a_{n}\right)\right) & =d\left(f_{m}\left(a_{m}\right), f_{m}(b)\right) \leq\left|a_{m}-b\right| \leq\left|a_{m}-a_{n}\right|+\left|a_{n}-b\right| \\
& \leq\left|a_{m}-a_{n}\right|+a_{m}^{m}-a_{n}^{n} .
\end{aligned}
$$

So $\left(f_{n}\left(a_{n}\right)\right)$ is a Cauchy sequence in $E$. Thus we can define $f: A \rightarrow \bar{E}$, where $\bar{E}$ is the completion of $E$, by setting, for $a \in A$,

$$
f(a)=\lim _{n \rightarrow \infty} f_{n}\left(a_{n}\right)
$$

where $\left(a_{n}\right)$ is a sequence such that $a_{n} \in A_{n}$ for all $n$ and $a_{n} \rightarrow a$ as $n \rightarrow \infty$. Clearly $f(a)$ does not depend on the choice of the sequence $\left(a_{n}\right)$. Let $a, b \in A$ and let $a_{n} \rightarrow a$ and $b_{n} \rightarrow b$ be such that $a_{n}, b_{n} \in A_{n}$ for all $n$. Then, since $f_{n}$ is 1-Lipschitz for each $n$,

$$
\begin{aligned}
d(f(a), f(b)) & \leq d\left(f(a), f_{n}\left(a_{n}\right)\right)+d\left(f_{n}\left(a_{n}\right), f_{n}\left(b_{n}\right)\right)+d\left(f_{n}\left(b_{n}\right), f(b)\right) \\
& \leq d\left(f(a), f_{n}\left(a_{n}\right)\right)+\left|a_{n}-b_{n}\right|+d\left(f_{n}\left(b_{n}\right), f(b)\right) \rightarrow|a-b|
\end{aligned}
$$

as $n \rightarrow \infty$. So $f$ is 1-Lipschitz. It is also surjective. To check this let $x \in D_{k}$ for some $k$. Then we have a sequence $\left(c_{n}\right)$ such that $c_{n} \in A_{n}$ and $f_{n}\left(c_{n}\right)=x$ for any $n \geq k$. Since the sequence $\left(c_{n}\right)_{n \geq k}$ is increasing and bounded, there is $c \in[0, L]$ such that $c_{n} \rightarrow c$. Then $c \in A$ by (i) and $x=\lim _{n \rightarrow \infty} f_{n}\left(c_{n}\right)=$ $f(c)$. Thus $D \subset f(A)$. Since $D \subset \bar{E}$ is dense and $f(A)$ is compact, we have $E \subset f(A)=\bar{E}$. Finally, we restrict $f$ to $f^{-1}(E)$.

TheOREM 5.3. Let $E$ be a bounded metric space such that $\beta(E)<\infty$. Then there exist $A \subset[0,1]$ and a Lipschitz surjection $f: A \rightarrow E$. Moreover, $f$ can be chosen such that $\operatorname{Lip}(f) \leq C(\beta(E)+d(E))$, where $C>0$ is an absolute constant. 
Proof. Let $\left(\Delta_{k}\right)_{k \in \mathbb{Z}}$ be a net of $E$ such that

$$
\sum_{k} \sum_{x \in \Delta_{k}} \beta\left(x, C_{2} 2^{-k}\right)^{2}\left(2^{-k}\right)^{3}<\infty
$$

and let $C_{3}, C_{4}$ and $\varepsilon_{0}$ be positive constants with $C_{3} \geq 9, C_{4}>24\left(1+C_{3}\right)$, $C_{2} \geq 4 C_{4}$ and $2 C_{4}\left(1+2 C_{4}\right) \varepsilon_{0} \leq \sqrt{4 C_{4}+1}$.

Suppose that $\beta\left(x, C_{2} 2^{-k}\right) 2^{-k}<\varepsilon \leq \sqrt{3} / 4$ for some $x \in D_{y, k}$, where $y \in E, k \in \mathbb{Z}$ and $D_{y, k}=B\left(y, 2^{-k+1}\right) \cap \Delta_{k}$. Since $C_{1} \geq C_{2} \geq 4$, we have $d\left(z_{1}, z_{2}\right) \geq C_{1}^{-1} C_{2} 2^{-k}$ for all $z_{1}, z_{2} \in D_{y, k} \subset B\left(x, C_{2} 2^{-k}\right)$. By (1),

$$
\begin{aligned}
\sin \varangle z_{1} z_{2} z_{3} & \leq \frac{d\left(D_{y, k}\right) \sin \varangle z_{1} z_{2} z_{3}}{d\left(z_{1}, z_{3}\right)} \leq \frac{d\left(D_{y, k}\right)}{2} c\left(z_{1}, z_{2}, z_{3}\right)<2^{k-1} d\left(D_{y, k}\right) \varepsilon \\
& \leq 2 \varepsilon \leq \frac{\sqrt{3}}{2}
\end{aligned}
$$

for any triple $\left\{z_{1}, z_{2}, z_{3}\right\} \subset D_{y, k}$. From this we conclude $D_{y, k} \in \Omega\left(\sqrt{1-4 \varepsilon^{2}}\right)$. If now $289\left(1-4 \varepsilon^{2}\right)^{3} \geq 225$, by Lemma 2.3 and Proposition 4.2 we have

$$
\left(\# D_{y, k}-1\right) 2^{-k} \leq \frac{d\left(D_{y, k}\right)}{\sqrt{1-4 \varepsilon^{2}}} \leq \frac{2^{-k+2}}{\sqrt{1-4 \varepsilon^{2}}},
$$

from which we get $\# D_{y, k} \leq 4 / \sqrt{1-4 \varepsilon^{2}}+1<6$. In particular, $\Delta_{k}$ is finite for each $k$. Let

$$
\bigcup_{k \in \mathbb{Z}} \Delta_{k}=\left\{x_{1}, x_{2}, x_{3}, \ldots\right\}
$$

so that for all $k \in \mathbb{Z}$,

$$
\begin{gathered}
D_{\# \Delta_{k}}=\Delta_{k}, \\
d\left(x_{j+1}, D_{j}\right)=\max \left\{d\left(x, D_{j}\right): x \in \Delta_{k}\right\} \quad \text { for } j=1, \ldots, \# \Delta_{k}-1,
\end{gathered}
$$

where $D_{j}=\left\{x_{1}, \ldots, x_{j}\right\}$ for $j \in \mathbb{N}$.

We are going to construct a sequence $\left(G_{j}\right)$ of connected weighted graphs with no cycles. For each $j$ we denote by $V_{j}$ and $E_{j}$ the sets of vertices and edges of $G_{j}$. For each $j$ we will have $D_{j} \subset V_{j}$. For all $x, y \in D_{j}$ such that $\{x, y\} \in E_{j}$ we will have $w_{j}(\{x, y\})=d(x, y)$, where $\left.w_{j}: E_{j} \rightarrow\right] 0, \infty[$ is the weight function on the graph $G_{j}$. We define $l\left(G_{j}\right)=\sum_{e \in E_{j}} w_{j}(e)$ and for $y \in D_{j}$ we will use the notation

$$
N_{j}(y)=\left\{z \in D_{j}:\{y, z\} \in E_{j}\right\} .
$$

Each vertex in $V_{j} \backslash D_{j}$ will have only one neighbour. Thus the subgraph of $G_{j}$ induced by $D_{j}$ will also be connected. We will denote this graph and the set of its edges by $G_{j}^{*}$ and $E_{j}^{*}$. In our construction the number $l\left(G_{j}^{*}\right)=\sum_{e \in E_{j}^{*}} w_{j}(e)$ will remain bounded, from which we get the final conclusion. 
We define a graph $G_{2}$ with 4 vertices and 3 edges as follows. Put $V_{2}=$ $\left\{x_{1}, x_{2}, b_{1}, b_{2}\right\}$, where $\left\{b_{1}, b_{2}\right\} \cap E=\emptyset$, and set $E_{2}=\left\{\left\{x_{1}, x_{2}\right\},\left\{x_{1}, b_{1}\right\}\right.$, $\left.\left\{x_{2}, b_{2}\right\}\right\}, w_{2}\left(\left\{x_{1}, x_{2}\right\}\right)=d\left(x_{1}, x_{2}\right)$ and $w_{2}\left(\left\{x_{i}, b_{i}\right\}\right)=C_{3} d\left(x_{1}, x_{2}\right)$ for $i=1,2$. Then

$$
l\left(G_{2}\right) \leq\left(1+2 C_{3}\right) d(E) .
$$

Let now $j \geq 2$ and assume by induction that we have constructed a graph $\left.G_{j}=\left(V_{j}, E_{j}\right), w_{j}: E_{j} \rightarrow\right] 0, \infty\left[\right.$ such that $D_{j} \subset V_{j}$. We also assume that $G_{j}$ has the following property:

$(*) \quad$ Let $y \in D_{j}$. If $d\left(y, z_{1}\right)<C_{4} d\left(x_{j+1}, D_{j}\right)$ and $d\left(z_{1}, z_{2}\right)<d\left(\left\{z_{1}, y, z_{2}\right\}\right)$ for all $z_{1}, z_{2} \in N_{j}(y)$, then there is $b \in V_{j} \backslash D_{j}$ such that $\{y, b\} \in E_{j}$.

We set $x=x_{j+1}$. Let $y$ be a nearest neighbour of $x$ in $D_{j}$ and let $k$ be the smallest integer such that $x \in \Delta_{k}$. In other words, $\# \Delta_{k-1} \leq j<\# \Delta_{k}$.

CASE 1: $\beta\left(x, C_{2} 2^{-k}\right) 2^{-k} \geq \varepsilon_{0}$. We set $V_{j+1}=V_{j} \cup\{x, b\}$, where $b \notin$ $V_{j} \cup E$, and define

$$
E_{j+1}=E_{j} \cup\{\{y, x\},\{x, b\}\}
$$

and $\left.w_{j+1}: E_{j+1} \rightarrow\right] 0, \infty[$ by setting

$$
w_{j+1}(e)= \begin{cases}d(y, x) & \text { for } e=\{y, x\}, \\ C_{3} d(y, x) & \text { for } e=\{x, b\}, \\ w_{j}(e) & \text { for } e \in E_{j} .\end{cases}
$$

Now $G_{j+1}$ has the property $(*)$ and

$$
\begin{aligned}
l\left(G_{j+1}\right)-l\left(G_{j}\right) & =\left(1+C_{3}\right) d(y, x) \leq\left(1+C_{3}\right) 2^{-(k-1)} \\
& \leq \frac{2\left(1+C_{3}\right)}{\varepsilon_{0}^{2}} \beta\left(x, C_{2} 2^{-k}\right)^{2}\left(2^{-k}\right)^{3} .
\end{aligned}
$$

For the remaining cases we assume that $\beta\left(x, C_{2} 2^{-k}\right) 2^{-k}<\varepsilon_{0}$.

CASE 2: There exists $z \in N_{j}(y)$ such that $C_{4} d(y, x) \leq d(y, z)$. We define $G_{j+1}$ as in Case 1. Now

$$
l\left(G_{j+1}\right)-l\left(G_{j}\right)=\left(1+C_{3}\right) d(y, x) \leq \frac{1+C_{3}}{C_{4}} d(y, z) .
$$

By the construction $\{y, z\} \in E_{m}^{*}$ for all $m \geq j$.

For the rest of the cases we assume that $d(y, z)<C_{4} d(y, x)$ for all $z \in N_{j}(y)$.

CASE 3: There exists $z \in N_{j}(y)$ such that $d(x, z) \leq d(y, z)$. We set $V_{j+1}=V_{j} \cup\{x\}$ and define

$$
E_{j+1}=\left(E_{j} \backslash\{\{y, z\}\}\right) \cup\{\{y, x\},\{x, z\}\}
$$


and $\left.w_{j+1}: E_{j+1} \rightarrow\right] 0, \infty[$ by setting

$$
w_{j+1}(e)= \begin{cases}d(y, x) & \text { for } e=\{y, x\} \\ d(x, z) & \text { for } e=\{x, z\} \\ w_{j}(e) & \text { for } e \in E_{j} \backslash\{\{y, z\}\} .\end{cases}
$$

By Lemma 5.1,

$$
\begin{aligned}
l\left(G_{j+1}\right)-l\left(G_{j}\right) & =d(y, x)+d(x, z)-d(y, z) \\
& \leq \frac{c(y, x, z)^{2}}{4} d(y, x) d(x, z)(d(y, x)+d(x, z)) \\
& \leq \frac{C_{4}\left(1+C_{4}\right)}{4} c(y, x, z)^{2}\left(2^{-(k-1)}\right)^{3} \\
& \leq 2 C_{4}\left(1+C_{4}\right) \beta\left(x, C_{2} 2^{-k}\right)^{2}\left(2^{-k}\right)^{3} .
\end{aligned}
$$

The last inequality holds, because $C_{1} \geq C_{2} \geq 2 C_{4}$.

We next show that $G_{j+1}$ has the property $(*)$ at $z$. Suppose that $\{z, b\} \notin$ $E_{j+1}$ for all $b \in V_{j+1} \backslash D_{j+1}$, which implies that $\{z, b\} \notin E_{j}$ for all $b \in V_{j} \backslash D_{j}$. Suppose further that $d(z, v)<C_{4} d\left(x_{j+2}, D_{j+1}\right)$ for all $v \in N_{j+1}(z)$. Then, since $d(y, z)<C_{4} d(y, x)$, we have $d(z, v)<C_{4} d\left(x_{j+1}, D_{j}\right)$ for all $v \in N_{j}(z)$. Thus by $(*)$ there exist $y^{\prime}, z^{\prime} \in N_{j}(z)$ for which $d\left(y^{\prime}, z^{\prime}\right)=d\left(\left\{y^{\prime}, z, z^{\prime}\right\}\right)$. If $y \notin\left\{y^{\prime}, z^{\prime}\right\}$, then $y^{\prime}, z^{\prime} \in N_{j+1}(z)$ and the property (*) is satisfied at $z$. Thus we may assume $y^{\prime}=y$. Now $2^{-k}<d(y, x) \leq 2^{-(k-1)}, d(x, z) \leq d(y, z)<$ $C_{4} d(y, x)$ and $\max \left\{d\left(y, z^{\prime}\right), d\left(x, z^{\prime}\right)\right\} \leq d(y, z)+d\left(z, z^{\prime}\right)<2 C_{4} d(y, x)$. Since $C_{1} \geq C_{2} \geq 4 C_{4}$ and $4 C_{4} \varepsilon_{0} \leq \sqrt{3}$, we have $\left\{y, x, z, z^{\prime}\right\} \in \Omega(\delta)$, where

$$
\delta \geq \sqrt{1-4 C_{4} \varepsilon_{0}^{2}} \geq \frac{2 C_{4}}{2 C_{4}+1} .
$$

Since now $y x z$ and $y z z^{\prime},\left\{y, x, z, z^{\prime}\right\}$ has an order by Lemma 2.2. Thus $d\left(x, z^{\prime}\right)=d\left(\left\{x, z, z^{\prime}\right\}\right)$ and $(*)$ is satisfied at $z$. Similarly we see that it is satisfied at $y$.

CASE 4: $d(y, z)<d(x, z)$ for all $z \in N_{j}(y)$. We first show that there exists $b \in V_{j} \backslash D_{j}$ such that $\{y, b\} \in E_{j}$. Suppose this fails. Now $d(y, v)<$ $C_{4} d(y, x)=C_{4} d\left(x_{j+1}, D_{j}\right)$ for all $v \in N_{j}(y)$. Thus by $(*)$ there are $z_{1}, z_{2} \in$ $N_{j}(y)$ such that $d\left(z_{1}, z_{2}\right)=d\left(\left\{z_{1}, y, z_{2}\right\}\right)$. Since $C_{1} \geq C_{2} \geq 2\left(1+C_{4}\right)$ and $4 C_{4} \varepsilon_{0} \leq \sqrt{3}$, we have $\left\{z_{1}, x, y, z_{2}\right\} \in \Omega(\delta)$, where

$$
\delta \geq \sqrt{1-4 C_{4}^{2} \varepsilon_{0}^{2}} \geq \frac{2 C_{4}}{2 C_{4}+1} .
$$

Since now $x y z_{1}$ and $x y z_{2}$, it follows from Lemma 2.2 that $y z_{1} z_{2}$ or $y z_{2} z_{1}$, which is a contradiction.

We set $V_{j+1}=V_{j} \cup\{x\}$ and define

$$
E_{j+1}=\left(E_{j} \backslash\{\{y, b\}\}\right) \cup\{\{y, x\},\{x, b\}\}
$$


and $\left.w_{j+1}: E_{j+1} \rightarrow\right] 0, \infty[$ by setting

$$
w_{j+1}(e)= \begin{cases}d(y, x) & \text { for } e=\{y, x\}, \\ w_{j}(\{y, b\}) & \text { for } e=\{x, b\} \\ w_{j}(e) & \text { for } e \in E_{j} \backslash\{y, b\} .\end{cases}
$$

Now

$$
l\left(G_{j+1}\right)-l\left(G_{j}\right)=d(y, x) \leq \frac{w_{j}(\{y, b\})}{C_{3}} .
$$

Since $d(x, z)=d(\{x, y, z\})$, the property $(*)$ is satisfied at $y$. For all $m \geq j$ there is $z \in D_{m}$ such that $\{z, b\} \in E_{m}$ and $w_{m}(\{z, b\})=w_{j}(\{y, b\})$ by the construction.

By iterating the above algorithm, we construct a sequence $\left(G_{j}\right)$ of graphs. Let $n_{0}$ be the smallest integer such that $\# \Delta_{n_{0}} \geq 2$. For all $n \geq n_{0}$ we define $T_{n}=G_{\# \Delta_{n}}^{*}$.

Since $289\left(1-4 \varepsilon_{0}^{2}\right)^{3} \geq 225$, for any $y \in E$ and $k$ Case 2 applies at most to four points in $B\left(y, 2^{-k+1}\right) \cap \Delta_{k}$ by the calculation at the beginning of the proof. Thus by (27) and the remark after it,

$$
\begin{aligned}
\sum_{j \in Y_{m}} \sum_{e \in E_{j} \backslash E_{j-1}} w_{j}(e) & \leq 2\left(1+\sum_{i=0}^{\infty} 2^{-i}\right) \frac{4\left(1+C_{3}\right)}{C_{4}} l\left(G_{m}^{*}\right) \\
& =\frac{24\left(1+C_{3}\right)}{C_{4}} l\left(G_{m}^{*}\right)
\end{aligned}
$$

for all $m \geq 3$, where $Y_{m}=\left\{j \in\{3, \ldots, m\}\right.$ : Case 2 applies to $\left.x_{j}\right\}$.

We now show that for any fixed $b \in \bigcup_{j}\left(V_{j} \backslash D_{j}\right)$ for all $k$ Case 4 can occur at most for three points in $\Delta_{k}$. Suppose this fails for some $k$ and let $\# \Delta_{k-1}<i_{1}<i_{2}<i_{3}<i_{4} \leq \# \Delta_{k}$ and $i_{0}<i_{1}$ be such that $\left\{x_{i_{l}}, b\right\} \in E_{i_{l}}$ for $l=0, \ldots, 4$. Now $x_{i_{l}} x_{i_{l+1}} x_{i_{l+2}}$ for $l=0,1,2$. Namely, if this is not true for some $l \in\{0,1,2\}$, there exists a nonempty set $\left\{y_{1}, \ldots, y_{p}\right\} \subset D_{i_{l+2}-1}$ such that $y_{p} x_{i_{l+1}} x_{i_{l+2}}, x_{i_{l}} y_{1} x_{i_{l+1}}$ and $y_{q} y_{q+1} x_{i_{l+1}}$ for $q=1, \ldots, p-1$. Since $2^{-k}<d\left(z_{1}, z_{2}\right) \leq 2^{-k+3}$ for all $z_{1}, z_{2} \in\left\{x_{i_{0}}, x_{i_{1}}, x_{i_{2}}, x_{i_{3}}, x_{i_{4}}, y_{1}, \ldots, y_{p}\right\} \subset$ $B\left(x_{i_{2}}, 2^{-k+2}\right), \beta\left(x_{i_{2}}, C_{2} 2^{-k}\right) 2^{-k}<\varepsilon_{0}, C_{1} \geq C_{2} \geq 4$ and $8 \varepsilon_{0} \leq \sqrt{3}$, we have $\left\{x_{i_{0}}, x_{i_{1}}, x_{i_{2}}, x_{i_{3}}, x_{i_{4}}, y_{1}, \ldots, y_{p}\right\} \in \Omega(\delta)$, where $\delta \geq \sqrt{1-16 \varepsilon_{0}^{2}}$. Since $\delta^{3} \geq 31 / 33,\left\{x_{i_{0}}, x_{i_{1}}, x_{i_{2}}, x_{i_{3}}, x_{i_{4}}, y_{1}, \ldots, y_{p}\right\}$ has an order by Lemma 2.3, from which we conclude $x_{i_{l}} x_{i_{l+1}} x_{i_{l+2}}$. Since $\max \left\{d\left(x, D_{i_{1}-1}\right): x \in \Delta_{k}\right\}$ $=d\left(x_{i_{1}}, x_{i_{0}}\right)<d\left(x_{i_{2}}, x_{i_{0}}\right)$, there is $z \in D_{i_{1}-1} \backslash\left\{x_{i_{0}}\right\}$ such that $d\left(x_{i_{2}}, z\right)$ $\leq d\left(x_{i_{1}}, x_{i_{0}}\right)$. As above, $\left\{x_{i_{0}}, x_{i_{1}}, x_{i_{2}}, x_{i_{3}}, x_{i_{4}}, z\right\}$ has an order by Lemma 2.3. Since $d\left(x_{i_{l}}, x_{i_{l-1}}\right)=d\left(x_{i_{l}}, D_{i_{l}-1}\right)$ for $l=1, \ldots, 4$, we must have $x_{i_{0}} x_{i_{1}} x_{i_{2}} x_{i_{3}} x_{i_{4}} z$. From this we get $d\left(x_{i_{2}}, z\right) \geq d\left(x_{i_{2}}, x_{i_{3}}\right)+\delta d\left(x_{i_{3}}, x_{i_{4}}\right)+$ $\delta d\left(x_{i_{4}}, z\right)>(1+2 \delta) 2^{-k}>2^{-(k-1)} \geq d\left(x_{i_{1}}, x_{i_{0}}\right)$, which is a contradiction. 
Thus by (29) and the remark after it,

$$
\begin{aligned}
\sum_{j \in Z_{m}} \sum_{e \in E_{j} \backslash E_{j-1}} w_{j}(e) & \leq\left(1+\sum_{i=0}^{\infty} 2^{-i}\right) \frac{3}{C_{3}}\left[l\left(G_{m}\right)-l\left(G_{m}^{*}\right)\right] \\
& =\frac{9}{C_{3}}\left[l\left(G_{m}\right)-l\left(G_{m}^{*}\right)\right]
\end{aligned}
$$

for all $m \geq 3$, where $Z_{m}=\left\{j \in\{3, \ldots, m\}\right.$ : Case 4 applies to $\left.x_{j}\right\}$.

Using the estimates (25), (26), (28), (30) and (31), we get for all $n \geq n_{0}$,

$$
\begin{aligned}
l\left(T_{n}\right) \leq & \left(1+2 C_{3}\right) d(E) \\
& +\max \left\{\frac{2\left(1+C_{3}\right)}{\varepsilon_{0}^{2}}, 2 C_{4}\left(1+C_{4}\right)\right\} \sum_{k=n_{0}}^{n} \sum_{x \in \Delta_{k} \backslash \Delta_{k-1}} \beta\left(x, C_{2} 2^{-k}\right)^{2}\left(2^{-k}\right)^{3} \\
& +\frac{24\left(1+C_{3}\right)}{C_{4}} l\left(T_{n}\right)+\frac{9}{C_{3}}\left[l\left(G_{\# \Delta_{n}}\right)-l\left(T_{n}\right)\right]-\left[l\left(G_{\# \Delta_{n}}\right)-l\left(T_{n}\right)\right] .
\end{aligned}
$$

Since $C_{3} \geq 9, C_{4}>24\left(1+C_{3}\right)$ and the net $\left(\Delta_{k}\right)_{k}$ is arbitrary, we have an absolute constant $C$ such that $2 l\left(G_{j}^{*}\right) \leq C(\beta(E)+d(E))$ for all $j \geq 2$. This means that for every $j$ we have a 1-Lipschitz surjection from $A_{j}$ to $D_{j}$, where $A_{j} \subset[0, C(\beta(E)+d(E))]$. Using Lemma 5.2 we get the result.

Acknowledgements. The author wishes to thank Professor Pertti Mattila for useful suggestions and for reading the manuscript.

\section{References}

[1] L. M. Blumenthal, Theory and Applications of Distance Geometry, Clarendon Press, Oxford, 1953.

[2] L. M. Blumenthal and K. Menger, Studies in Geometry, W. H. Freeman, 1970.

[3] G. David, Unrectifiable 1-sets have vanishing analytic capacity, Rev. Mat. Iberoamericana 14 (1998), 369-478.

[4] G. David and S. Semmes, Singular integrals and rectifiable sets in $\mathbb{R}^{n}$, Astérisque 193 (1991).

[5] P. W. Jones, Rectifiable sets and the traveling salesman problem, Invent. Math. 102 (1990), 1-15.

[6] J.-C. Léger, Rectifiability and Menger curvature, Ann. of Math. 149 (1999), 831-869.

[7] P. Mattila, M. S. Melnikov and J. Verdera, The Cauchy integral, analytic capacity, and uniform rectifiability, Ann. of Math. 144 (1996), 127-136.

[8] M. S. Melnikov, Analytic capacity: discrete approach and curvature of measure, Sb. Math. 186 (1995), 827-846.

[9] K. Menger, Untersuchungen über allgemeine Metrik, Math. Ann. 100 (1928), 75-163.

[10] - Untersuchungen über allgemeine Metrik. Vierte Untersuchung. Zur Metrik der Kurven, ibid. 103 (1930), 466-501.

[11] K. Okikiolu, Characterization of subsets of rectifiable curves in $\mathbb{R}^{n}$, J. London Math. Soc. 46 (1992), 336-348. 
[12] I. J. Schoenberg, On metric arcs of vanishing Menger curvature, Ann. of Math. 41 (1940), 715-726.

[13] X. Tolsa, Painlevé's problem and the semiadditivity of analytic capacity, Acta Math. 190 (2003), 105-149.

Department of Mathematics and Statistics

University of Jyväskylä

P.O. Box 35

FIN-40014 Jyväskylä, Finland

E-mail: imhahlom@maths.jyu.fi

Received 21 June 2004;

in revised form 29 November 2004 